\title{
Structure-activity relationships in platelet-activating factor. Part 14: Synthesis and biological evaluation of piperazine derivatives with dual anti-PAF and anti-HIV-1 activity
}

\author{
Wafa Sallem, ${ }^{\mathrm{a}}$ Nawal Serradji, ${ }^{\mathrm{a}, *}$ Nathalie Dereuddre-Bosquet, ${ }^{\mathrm{b}}$ Georges Dive, ${ }^{\mathrm{c}}$ \\ Pascal Clayette ${ }^{\mathrm{b}}$ and Françoise Heymans ${ }^{\mathrm{a}}$ \\ ${ }^{a}$ Unité de Recherche Pharmacochimie Moléculaire et Systèmes Membranaires (EA 2381), \\ Laboratoire de Pharmacochimie Moléculaire, Université Paris 7 - Denis Diderot, case 7066, \\ 2 Place Jussieu, 75251 Paris Cedex 05, France \\ ${ }^{\mathrm{b}}$ SPI-BIO, CEA, DSVIDRM, 18 route du Panorama, BP 6, 92265 Fontenay aux Roses Cedex, France \\ ${ }^{\mathrm{c}}$ Centre d'Ingénierie des Protéines, Institut de Chimie, Université de Liège, Bâtiment B6, Allée du 6 Août, \\ 13 Sart Tilman, 4000 Liège, Belgium
}

Received 26 June 2006; revised 20 July 2006; accepted 24 July 2006

\begin{abstract}
As HIV-associated dementia prevalence has risen with the lifespan of HIV-infected individuals, there is an important need for antiretroviral and anti-inflammatory drugs targeting the central nervous system. Platelet-activating factor, a mediator of inflammation, is an HIV-induced neurotoxin secreted in the infected brain. In this work, we developed piperazine derivatives bearing a heterocyclic moiety as PAF-antagonists and HIV-1 replication inhibitors with micromolar potency.

(c) 2006 Elsevier Ltd. All rights reserved.
\end{abstract}

\section{Introduction}

The infection of central nervous system (CNS) by human immunodeficiency virus-1 (HIV-1) occurs early after the systemic infection. ${ }^{1,2}$ Mild to severe neurological complications as a consequence of this infection are known as HIV-associated dementia (HAD) or acquired immunodeficiency syndrome (AIDS) dementia complex (ADC) and were observed in about $30 \%$ of adults and half of children with AIDS before the use of highly active antiretroviral therapy (HAART) in the developed world. ${ }^{3,4}$ HAART has played an important role in declining the incidence of neurological complications in AIDS patients (approximately 10\% now) ${ }^{5,6}$ but these neurological impairments still occur not only in untreated but in treated individuals as well and HAD prevalence has risen with HIV-infected individual's lifespan as they live longer. ${ }^{7-9}$ Moreover, with the use of HAART, minor cognitive motor disorder (MCMD), a

\footnotetext{
Keywords: Piperazine; Anti-PAF; Anti-HIV-1; AIDS dementia complex.

* Corresponding author. Tel.: +33 14427 5594; fax: +33 14427

5641; e-mail: serradji@paris7.jussieu.fr
}

less severe form of CNS dysfunction which does not always progress to $\mathrm{HAD}$, becomes more frequent ${ }^{10,11}$ and probably results from a weak penetration of the antiretroviral drugs into the $\mathrm{CNS}^{12}$ allowing a low-level viral replication and a progressive neurodegeneration. The pathogenesis of HAD may result from the secretion of neurotoxins by mononuclear phagocytes (MPs) (perivascular and brain macrophages and microglia) after HIV-1 infection, stimulation by viral proteins and immune activation. ${ }^{11,13}$ Platelet-activating factor (PAF), a phospholipid inflammatory mediator, is produced in response to the viral infection and activation of MPs and has been identified as an HIV-1-induced neurotoxin. ${ }^{14,15}$ Indeed, HIV-1 production is up-regulated by TNF- $\alpha$ in infected macrophages, and PAF, in turn, appears to increase TNF- $\alpha$ synthesis in HIV-infected cells of monocytic lineage. ${ }^{16,17}$ High levels of PAF are detected in cerebrospinal fluids (CSF) of patients with neurologic dysfunctions and correlated with the degree of neurological impairments. Moreover, when added at concentrations close to those found in CSF of HIV-infected patients, PAF produces a dose-dependent neurotoxicity, inhibited by NMDA antagonists MK-801 and memantine. ${ }^{14}$ The neurotoxicity induced by HIV-1-infected macrophages can be prevented by 

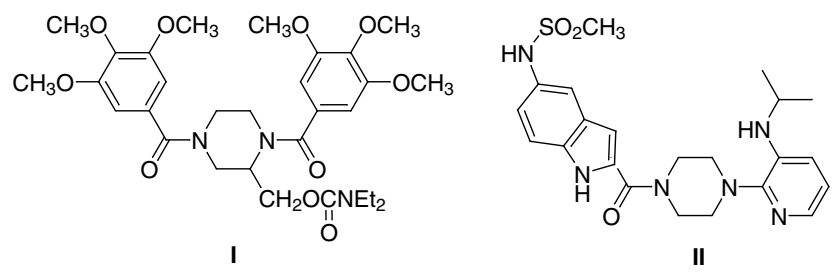

Figure 1. PMS 601 (I) and Delavirdine (II) structures.

PAF-acetylhydrolase (PAF-AH), the main enzyme responsible for PAF catabolism, or WEB 2086, a PAF receptor antagonist. ${ }^{15}$ Taking together, these results suggest that PAF plays an important role in the HIV-1-induced neurologic dysfunctions. These findings prompted us to evaluate, in a previous work, the antiviral activity of several PAF-antagonists and led us to the discovery of a lead compound, PMS 601 (Fig. 1), a trisubstituted piperazine with both a PAF-antagonism $\left(\mathrm{IC}_{50}=8 \mu \mathrm{M}\right)$ and anti-HIV-1 effects $\left(\mathrm{IC}_{50}=11 \mu \mathrm{M}\right) .{ }^{18}$ All the modifications undertaken in order to improve its dual activity have been realized by either replacing the carbamate group of PMS 601 by other functions ${ }^{19}$ or introducing an aromatic group on one or both nitrogens of the piperazine ring. ${ }^{20}$ Those studies showed that more potent compounds than PMS 601 can be obtained and denote the absence of correlation between both anti-PAF and anti-HIV activities. Moreover, PMS 601 and Delavirdine (Rescriptor ${ }^{\circledR}$, Fig. 1), a nonnucleoside reverse transcriptase inhibitor, are both piperazine derivatives carrying aryl groups as nitrogen substituents. This observation prompted us to replace one of both trimeth- oxyphenyl groups of PMS $\mathbf{6 0 1}$ by an indole moiety found in Delavirdine and to evaluate the influence of this modification on both biological activities. We described here the structure-activity relationship triggered around this heterocyclic moiety by (i) varying the nature of the linker between this ring and the piperazine and its position on the indole group; (ii) introducing an electron-donating or -withdrawing group on $\mathrm{C}_{5}$-indole; (iii) incorporating other heterocyclic units; (iv) methylating the indole nitrogen. Moreover, the second trimethoxybenzoyl substituent of PMS 601 was replaced by a benzyl or a 3,4,5-trimethoxybenzyl group since a previous study showed that compounds with a benzylic moiety exhibit higher antiviral activities than our reference compound. ${ }^{20}$ All compounds were tested in vitro to evaluate their anti-PAF and anti-HIV-1 activities. The $3 \mathrm{D}$ electrostatic potential maps of certain compounds were also calculated to understand their anti-PAF activity.

\section{Results and discussion}

\subsection{Chemistry}

In Scheme 1, the monoacylated piperazines $\mathbf{1}$ and $\mathbf{2}$ were prepared according to the previously published procedures. ${ }^{20}$ Coupling $\mathbf{1}$ and $\mathbf{2}$ with commercial heterocyclic acid $(\mathrm{HetCOOH})$, in the presence of DCC or DIC, led to the heteroarylamide piperazine derivatives $\mathbf{3 a}-\mathbf{j}$ and $\mathbf{4 a}-\mathbf{f}$. The $\mathrm{N}$-methylation of $\mathbf{3 a}$ and $\mathbf{4 a}$ using iodomethane and $\mathrm{K}_{2} \mathrm{CO}_{3}$ in acetonitrile gave $3 \mathbf{k}$ and $\mathbf{4 k}$, respectively. The compounds $\mathbf{6 a}, \mathbf{b}$ were obtained, as outlined

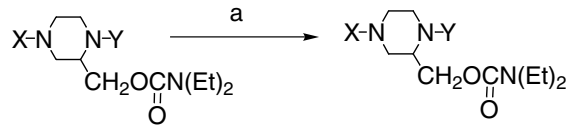

1: $\mathrm{X}=\mathrm{TMB} ; \mathrm{Y}=\mathrm{H}$ 2: $\mathrm{X}=\mathrm{H} ; \mathrm{Y}=\mathrm{TMB}$

3a-j: $X=$ TMB ; $Y=$ Het b $4 a-f: X=H e t ; Y=T M B$
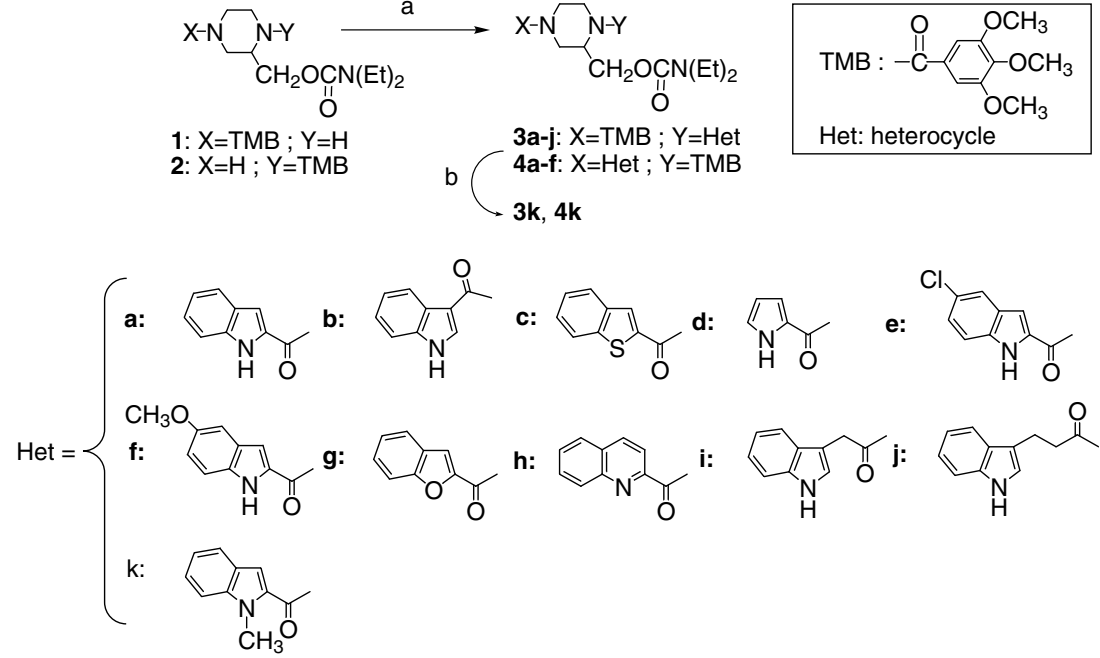

Scheme 1. Reagents and conditions: (a) $\mathrm{HetCOOH}$, DCC or DIC, $\mathrm{HOBT}, \mathrm{CH}_{2} \mathrm{Cl}_{2}$, reflux; (b) $\mathrm{CH}_{3} \mathrm{I}, \mathrm{K}_{2} \mathrm{CO}_{3}, \mathrm{CH}_{3} \mathrm{CN}$, reflux.
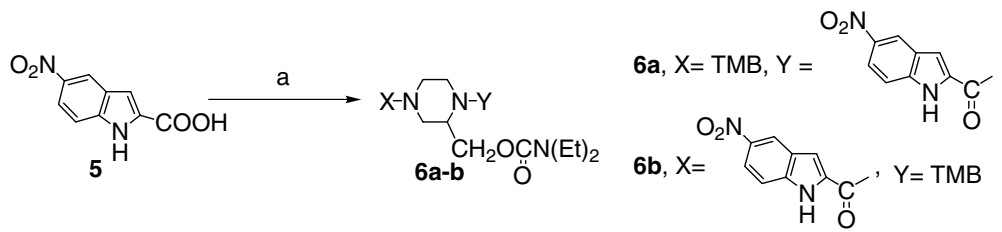

Scheme 2. Reagents: (a) $\mathrm{i}-\mathrm{SOCl}_{2}, \mathrm{CH}_{2} \mathrm{Cl}_{2}$; ii-1 or 2, $\mathrm{Et}_{3} \mathrm{~N}, \mathrm{CH}_{2} \mathrm{Cl}_{2}$. 
in Scheme 2, from the acyl chloride of 5-nitro- $1 H$-indole-2-carboxylic acid $\mathbf{5},{ }^{21}$ prepared in situ using thionyl chloride, and the piperazine derivatives $\mathbf{1}$ and $\mathbf{2}$, respectively. The compound $\mathbf{8}$ was synthesized, as shown in Scheme 3, by reaction of the amine 1 with $7 .^{22}$ Treatment of $9^{23}$ with DIC led to indole-1-carboxylic anhydride which was coupled directly to the amine 1 to provide 10 (Scheme 4). The synthetic route to obtain the derivatives 16a,b, 17a, b and 18 is detailed in Scheme 5. The condensation of $\mathbf{1 1}{ }^{20}$ with $1 H$-indole-2-carboxylic acid led to 12 which was deprotected into 13 using trifluoroacetic acid (TFA) in $\mathrm{MeOH}$. The monosubstitution of $14^{24}$ using $1 H$-indole-2-carboxylic acid and DCC led to 15. The compounds $16 \mathbf{a}, \mathbf{b}, \mathbf{1 7 a}, \mathbf{b}$ and 18 were obtained using a reductive amination process from the piperazines 13, 15 and 2, respectively, and the corresponding aldehyde in dichloromethane or dichloroethane (DCE).

\subsection{Anti-PAF and anti-HIV effects}

All final compounds were tested for their ability to antagonize PAF-induced platelet aggregation on the

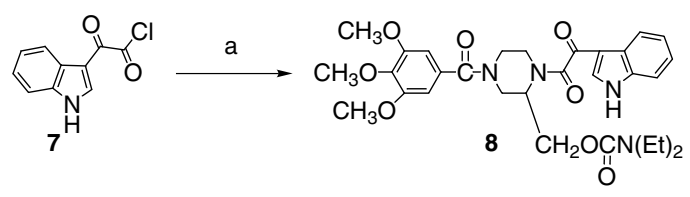

Scheme 3. Reagents: (a) $1, \mathrm{Et}_{3} \mathrm{~N}, \mathrm{CH}_{2} \mathrm{Cl}_{2}$. one hand and to inhibit the viral replication in MDM in vitro infected with the reference macrophage-tropic HIV-1/Ba-L strain on the other hand. ${ }^{18-20}$ All the results obtained are listed in Tables 1-3. Our initial synthetic efforts focused on determining the influence of the heterocyclic substitution position on the dual activity.

2.2.1. Anti-HIV activity (Tables 1-3). The anti-HIV target of this piperazinic series of compounds remains undefined. ${ }^{18}$ In this new set of experiments, PMS 601 and all the new compounds were tested according to the previously published procedures. ${ }^{18}$ The replacement of a 3,4,5-trimethoxylphenyl moiety of PMS $\mathbf{6 0 1}$ by an indole as in 3a (Table 1) leads to an activity close to the reference compound one. As a general trend for the results listed in Table 1, no real improvement of the antiviral activity is obtained when either the position of the carbonyl function on the indole group ( $\mathbf{3 a}, \mathbf{b}$, and 10) or the nature of the linker between this heterocycle and the piperazine ring $(\mathbf{3 i}, \mathbf{j}, \mathbf{8})$ is modified. The nature of the introduced heterocycle $(\mathbf{3} \mathbf{c}, \mathbf{d}, \mathbf{g}, \mathbf{h})$ or the electron donating or -withdrawing effects of the group on the $\mathrm{C}_{5}$-indole (3e,f, 6a) do not increase this activity as well. Nevertheless, a loss of activity is observed when the indole is replaced by another heterocyclic ring as benzofurane (3g), benzothiophene (3c), pyrrole (3d) and quinoleine (3h) or when it is $\mathrm{N}$-methylated (3k) suggesting that this heterocycle found in Delavirdine induces this activity. The comparison of Tables 1 and 2, where are listed the results obtained with the position analogs of the compounds listed in Table 1, shows that whatever

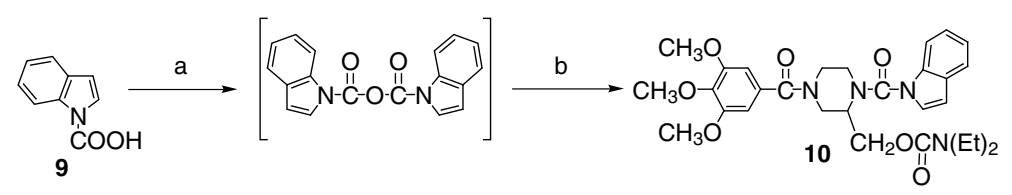

Scheme 4. Reagents: (a) DIC, $\mathrm{CH}_{2} \mathrm{Cl}_{2}$; (b) $\mathbf{1}, \mathrm{CH}_{2} \mathrm{Cl}_{2}$.
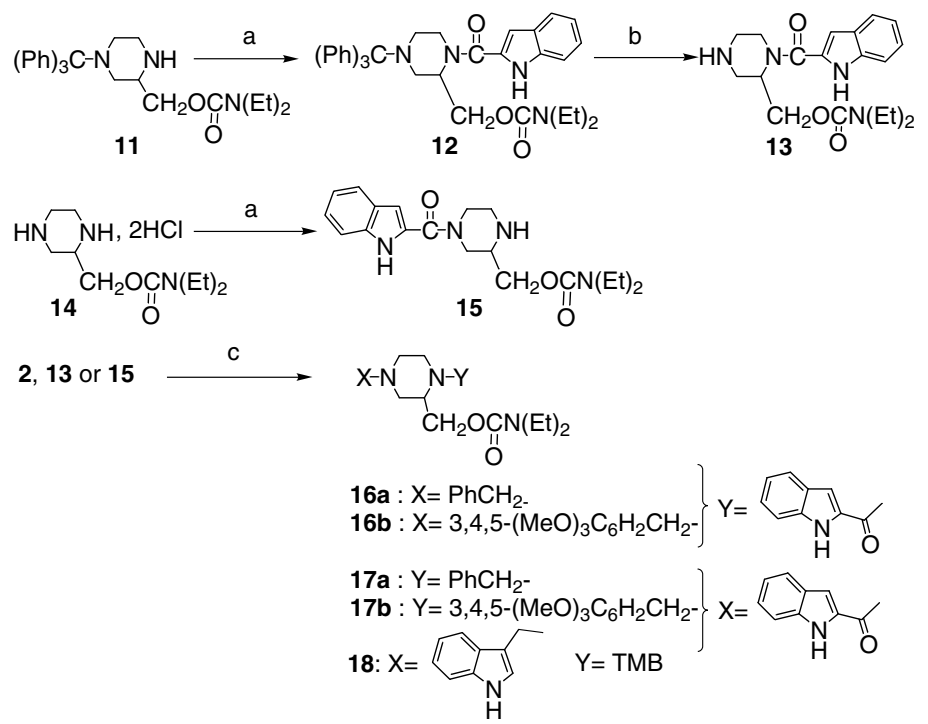

Scheme 5. Reagents and condition: (a) $1 \mathrm{H}$-indole-2-carboxylic acid, $\mathrm{DCC}, \mathrm{CH}_{2} \mathrm{Cl}_{2}$, reflux; (b) TFA, $\mathrm{MeOH}$; (c) $\mathrm{PhCHO}, 3,4,5-(\mathrm{MeO})_{3} \mathrm{C}_{6} \mathrm{H}_{2} \mathrm{CHO}$ or indole-3-carboxaldehyde, $\mathrm{NaBH}(\mathrm{OAc})_{3}, \mathrm{CH}_{2} \mathrm{Cl}_{2}$ or DCE. 
Table 1. In vitro anti-PAF and anti-HIV-1 activities of heterocyclic derivatives at the N1-position of the piperazine

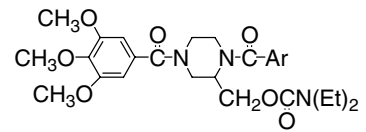

\begin{tabular}{|c|c|c|c|c|c|c|}
\hline \multirow[t]{2}{*}{ Compound } & \multirow[t]{2}{*}{$\mathrm{Ar}$} & \multirow[t]{2}{*}{ Anti-PAF $\mathrm{IC}_{50}(\mu \mathrm{M})$} & \multicolumn{3}{|c|}{ Anti-HIV $(\mu \mathrm{M})$} & \multirow[t]{2}{*}{ Toxicity $(\mu \mathrm{M})$} \\
\hline & & & $\mathrm{ED}_{50}$ & $\mathrm{ED}_{70}$ & $\mathrm{ED}_{90}$ & \\
\hline PMS 601 & & 8 & 32 & 90 & 480 & $>500$ \\
\hline $3 a$ & & 1 & 18.8 & 54 & $>100$ & 500 \\
\hline $3 b$ & & 24 & 4.5 & 17 & 140 & $>500$ \\
\hline $3 c$ & & 0.13 & 58 & 67 & 82 & 500 \\
\hline 3d & & 2.97 & & $-15 \pm 33 \%^{\mathrm{a}}$ & & $>50$ \\
\hline $3 e$ & & 0.28 & 7.3 & 13 & $>20$ & 100 \\
\hline $3 f$ & & 0.57 & 6 & 20 & 100 & 500 \\
\hline $3 g$ & & 0.5 & 97 & 130 & 210 & $>500$ \\
\hline $3 \mathrm{~h}$ & & 0.92 & & $36 \pm 9 \%{ }^{\mathrm{a}}$ & & $>50$ \\
\hline $3 \mathbf{i}$ & & 0.27 & 28 & 42 & 78 & $>500$ \\
\hline $3 \mathbf{j}$ & & 0.95 & 25 & 38 & 72 & 500 \\
\hline $3 k$ & & 25.50 & 58 & 100 & $>100$ & 500 \\
\hline $6 a$ & & 0.34 & & - & & $50^{\mathrm{b}}$ \\
\hline 8 & & 0.11 & 17 & $>100$ & $>100$ & 500 \\
\hline 10 & & 0.56 & 5.9 & 19 & $>20$ & 100 \\
\hline
\end{tabular}

a $\%$ of inhibition of viral replication at $50 \mu \mathrm{M}$.

${ }^{\mathrm{b}} 53 \pm 7 \%$ of toxicity at $50 \mu \mathrm{M}$.

be the position ( 1 or 4 ) of the heterocyclic moiety on the piperazine ring, the same range of activity is obtained (3a-f, $6 \mathbf{a}$ and $3 \mathbf{k}$ vs $4 \mathbf{a}-\mathbf{f}, \mathbf{6} \mathbf{b}$ and $\mathbf{4 k}$, respectively). A previous work underlined the positive effects of the introduction of a benzyl or a 3,4,5-trimethoxybenzyl group instead of a 3,4,5-trimethoxybenzoyl moiety on the antiviral activity (Table 3, compounds $\mathbf{A}$ and $\mathbf{B}$ ). ${ }^{20}$ To see whether this observation was available in the heterocyclic series, we synthesized the compounds listed in Table 3. All bear an indole and a benzyl or a 3,4, 
Table 2. In vitro anti-PAF and anti-HIV-1 activities of heterocyclic derivatives at the N4-position of the piperazine

\begin{tabular}{|c|c|c|c|c|c|c|}
\hline \multirow[t]{2}{*}{ Compound } & \multirow[t]{2}{*}{$\mathrm{Ar}$} & \multirow[t]{2}{*}{ Anti-PAF $\mathrm{IC}_{50}(\mu \mathrm{M})$} & \multicolumn{3}{|c|}{ Anti-HIV $(\mu \mathrm{M})$} & \multirow[t]{2}{*}{ Toxicity $(\mu \mathrm{M})$} \\
\hline & & & $\mathrm{ED}_{50}$ & $\mathrm{ED}_{70}$ & $\mathrm{ED}_{90}$ & \\
\hline $4 a$ & & 0.47 & 13 & 23.5 & 49 & 500 \\
\hline $4 b$ & & 5 & 6.5 & 16.1 & 75 & 500 \\
\hline $4 c$ & & 0.13 & & $15 \pm 17^{\mathrm{a}}$ & & 0 \\
\hline $4 d$ & & 1.32 & & $4 \pm 23^{\mathrm{a}}$ & & 0 \\
\hline $4 e$ & & 0.11 & 2.3 & 8.3 & $>20$ & 100 \\
\hline $4 f$ & & 0.017 & 37 & 56 & 100 & 500 \\
\hline $4 k$ & & 1 & 19 & 64 & $>100$ & 500 \\
\hline $\mathbf{6 b}$ & & 0.10 & & - & & $50^{\mathrm{b}}$ \\
\hline 18 & & 306 & & - & & $50^{\mathrm{c}}$ \\
\hline
\end{tabular}

\footnotetext{
$\mathrm{a}_{\%}$ of inhibition of viral replication at $50 \mu \mathrm{M}$.

${ }^{\mathrm{b}} 55 \pm 6 \%$ of toxicity at $50 \mu \mathrm{M}$.

c $89 \pm 2 \%$ of toxicity at $50 \mu \mathrm{M}$.
}

5-trimethoxybenzyl group on the piperazine. All the compounds are either inactive $(\mathbf{3 d}, \mathbf{4 c}-\mathbf{d})$, weakly active (3h) or cytotoxic $(6 \mathbf{a}, \mathbf{b}, 16 \mathbf{a}, \mathbf{b}, 17 \mathbf{a}, \mathbf{b}, 18)$ at the first dose tested, that is, $50 \mu \mathrm{M}$, suggesting that the introduction of a benzyl moiety in this heterocyclic series is not favourable to the antiviral activity.

2.2.2. Anti-PAF activity (Tables 1-3) and molecular modelling (Fig. 2). In this study, the geometry of the studied compounds has been fully optimized using the double basis set $6-31 \mathrm{gg}^{25}$

In 1991, Batt et al. proposed a model for the PAF-receptor (PAF-R) structure from 3D electrostatic maps of several structurally unrelated synthetic and natural PAF-antagonists. ${ }^{26}$ It would be a multipolarized cylinder with a diameter of $14 \AA$ with three couples of positive areas detected at $14,10-12$ and $6.5-7 \AA$ and would contain two hydrophobic subsites. In 1993, the calculations of 3D electrostatic maps of 3,4,5di(trimethoxybenzoyl)piperazine derivatives revealed that negative electrostatic potentials able to interact with the positive zones of the PAF-R are generated by, on the one hand, the methoxyl groups and form the large 'cache-oreilles' system (11-14 $\AA$ ) and by the carbonyl groups of the amide functions on the other hand and form the short 'cache-oreilles' system (6$7 \AA){ }^{24}$ Those potentials are determinant to the antiPAF activity. In this paper, we determined the $3 \mathrm{D}$ electrostatic potential maps (isocontoured at $-20 \mathrm{kcal} / \mathrm{mol}$ ) of PMS 601 and some heterocyclic derivatives in order to explain the anti-PAF activity obtained for those compounds. The PMS 601 3D electrostatic map (Fig. 2) presents two major 'cache-oreilles' systems generated by both the methoxyl groups, located at a distance of $11.34 \AA$, and the carbonyl groups of amides at a distance of $6.3 \AA$. This result is in harmony with what was previously established. The results presented in Table 1 show that the replacement of a 3,4,5-trimethoxyphenyl moiety of PMS 601 by an indole leads to the same anti-PAF activity. We can notice that whatever the nature of the substituent on the $\mathrm{C}_{5}$-indole (3e-f, $\left.\mathbf{6 a}\right)$, the linker between this heterocycle and the piperazine ring $(\mathbf{3 i}-\mathbf{j}, \mathbf{8})$, the connection position of the carbonyl to the indole ring and 
Table 3. In vitro anti-PAF and anti-HIV-1 activities of indole derivatives

\begin{tabular}{|c|c|c|c|c|c|}
\hline Compound & $\mathrm{Ar}_{1}$ & $\mathrm{Ar}_{2}$ & Anti-PAF $\mathrm{IC}_{50}(\mu \mathrm{M})$ & $\begin{array}{l}\text { Inhibition of viral } \\
\text { replication } \% \text { at } 50 \mu \mathrm{M}\end{array}$ & $\begin{array}{l}\text { Toxicity } \% \\
\text { at } 50 \mu \mathrm{M}\end{array}$ \\
\hline $\mathbf{A}^{\mathrm{a}}$ & & & 2.5 & 75 at $10 \mu \mathrm{M}^{\mathrm{b}}$ & 0 \\
\hline $\mathbf{B}^{\mathrm{a}}$ & & & $>100$ & $2^{\mathrm{c}}$ & $0^{\mathrm{d}}$ \\
\hline $\mathbf{C}^{\mathrm{a}}$ & & & 13 & $40^{\mathrm{c}}$ & 0 \\
\hline $\mathbf{D}^{\mathrm{a}}$ & & & $>100$ & $13^{\mathrm{c}}$ & 0 \\
\hline $3 \mathbf{a}$ & & & 1 & $70 \pm 9$ & 0 \\
\hline $4 a$ & & & 0.47 & $\begin{array}{l}46 \pm 28 \text { at } 20 \mu \mathrm{M} \\
93 \pm 3 \text { at } 100 \mu \mathrm{M}^{\mathrm{b}}\end{array}$ & 0 \\
\hline $16 a$ & & & 4.54 & - & $60 \pm 16$ \\
\hline $16 b$ & & & 0.12 & - & $82 \pm 4$ \\
\hline $17 \mathrm{a}$ & & & 157 & - & $85 \pm 3$ \\
\hline $17 b$ & & & 11 & - & $67 \pm 1$ \\
\hline
\end{tabular}

${ }^{a}$ Serradji et al. (Bioorg. Med. Chem., 2006).

${ }^{\mathrm{b}}$ Not performed at $50 \mu \mathrm{M}$ not provided.

${ }^{\mathrm{c}} \mathrm{ED}_{50}$.

${ }^{\mathrm{d}}$ Toxic at $100 \mu \mathrm{M}$.

the heterocyclic cycle introduced to replace the indole moiety $(\mathbf{3 c}-\mathbf{d}, \mathbf{g}-\mathbf{h})$, anti-PAF activity remains close to the micromolar range (except for $\mathbf{3 b}$ and $\mathbf{3 k}$ ).

The study of 3a electrostatic map shows that only the short 'cache-oreilles' system, generated by both carbonyl functions carried by the piperazine, is present in this molecule and seems to be responsible for the activity. This short system is also found in the compounds $\mathbf{1 0}$ (map not provided) and $\mathbf{3 b}$ although the latter is less active than 3a. Its 3D map reveals that the indole adopts a different spatial orientation from that observed in $\mathbf{3 a}$, suggesting an influence of the position of the carbonyl function on the molecule conformation which can be considered as a less active conformation in this case. Interestingly, the insertion of one methylene between the carbonyl and the indole in $\mathbf{3 i}$ confers a micromolar activity to the compound likely due to the freedom recovery of the indole ring orientation allowed by this methylene. Nevertheless, increasing this flexibility with one more methylene as in $\mathbf{3} \mathbf{j}$ (map not provided) does not improve the anti-PAF potency. The introduction of a second carbonyl in $\mathbf{8}$ pronounces the electronegative well around both carbonyls and then ameliorates the anti-PAF activity compared to $\mathbf{3 b}$. The introduction of an electron-donating (chloro in $\mathbf{3 e}$, map not provided, or methoxy in 3f) or -withdrawing (nitro in 6a, map not provided) substituent at the $\mathrm{C}_{5}$-position of the indole enables the formation of a supplementary negative electrostatic potential around this group, as shown in 3f map. Coupled to the well formed by the 3,4,5trimethoxyphenyl, it could be considered as the large 'cache-oreilles' system and would increase the activity of these compounds. Surprisingly, the compounds $\mathbf{3 e , f}$ and $\mathbf{6 a}$ are not more potent than 3a suggesting that either the potential created is insufficient or is not 


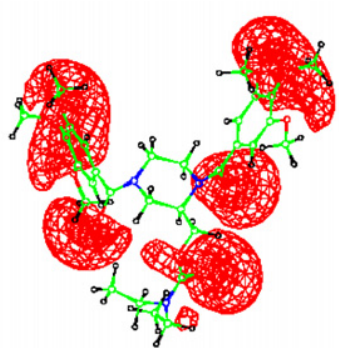

PMS 601

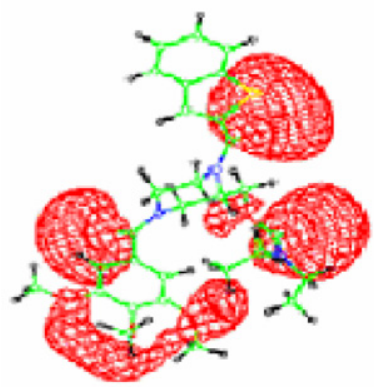

$3 \mathbf{c}$

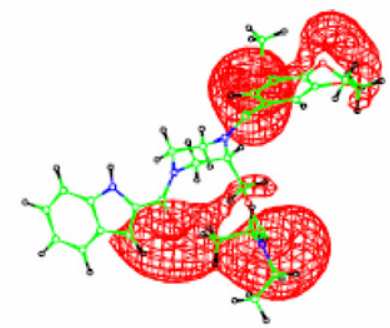

$4 \mathbf{a}$

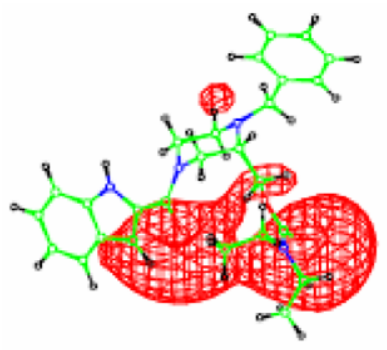

16a

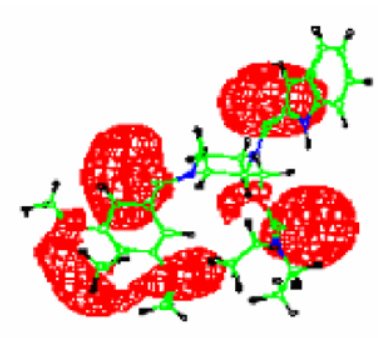

3a

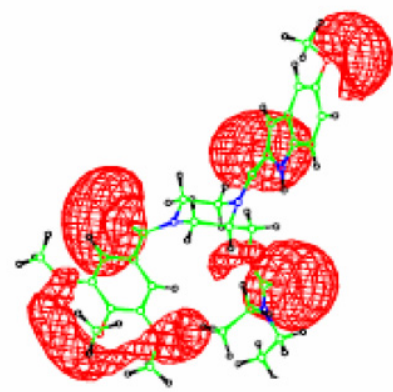

3f

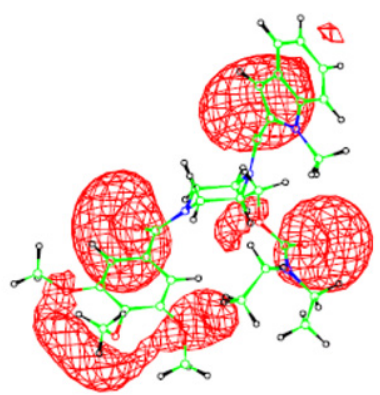

$3 \mathbf{k}$

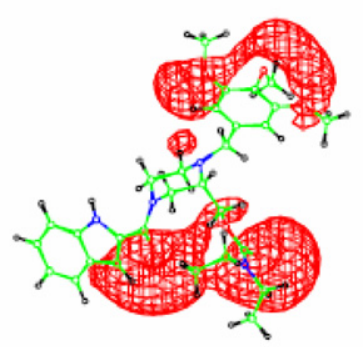

16b

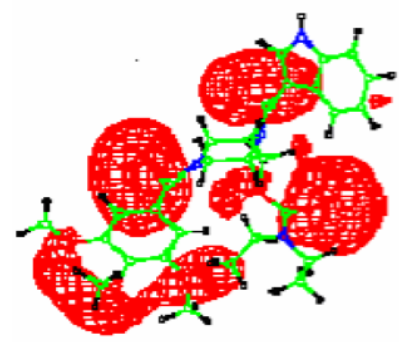

3b

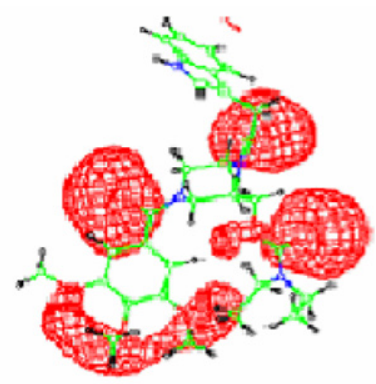

$3 \mathbf{i}$

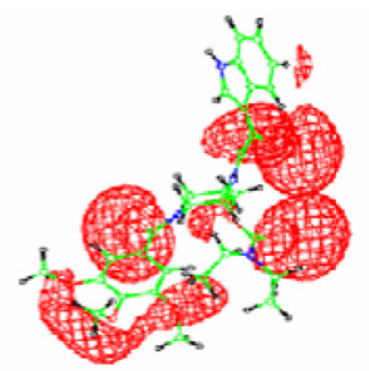

8

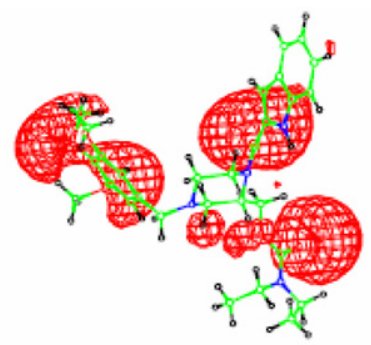

17b

Figure 2. 3D Electrostatic maps calculated of selected compounds.

located at the proper position, that is, at $10-14 \AA$ from the negative potential of the trimethoxyl groups. The replacement of the indole moiety by another heterocyclic ring (benzofuran in $\mathbf{3 g}$, benzothiophene in $\mathbf{3 c}$, pyrrole in 3d, and quinoline in $\mathbf{3 h}$ ) has no or little influence on the negative electrostatic potential generated by the carbonyl function as illustrated in the map of the compound $\mathbf{3 c}$ and thus does not greatly improve the anti-PAF activity of $\mathbf{3 a}$. The weak activity of $\mathbf{3 k}\left(\mathrm{IC}_{50}\right.$ of $25.5 \mu \mathrm{M}$ ) is due neither to an influence of the methyl on the conformation of the molecule nor to a modification of the carbonyl electrostatic potential through a H-bond as shown in Fig. 2. In Table 2 are summarized the activities obtained with the position isomers of several compounds in Table 1 and no significant influence of isomerization on the PAF-antagonism is observed (except for $\mathbf{4 b}, \mathbf{4 f}$ and $\mathbf{4 k}$ ). The compound $\mathbf{1 8}$, where the carbonyl of $\mathbf{4 b}$ was replaced by a methylene, is inactive as expected. Indeed, this modification deletes the potential induced by the carbonyl function and underlines the necessity of its presence for anti-PAF activity (map not provided). In Table 3, the replacement of the second tri- 
methoxybenzoyl moiety of 3a by a benzyl group as in 17a leads to a loss of activity because of the absence of a carbonyl function and its associated electrostatic potential. Surprisingly, its position analogue 16a is much more active. This difference of behaviour has already been observed in a previous work with the compounds $\mathbf{C}$ and $\mathbf{D}$ where a benzyl group is present as well and should be clarified. The introduction of a 3,4,5-trimethoxybenzyl moiety in $\mathbf{1 6 b}$ and $\mathbf{1 7 b}$ confers an anti-PAF potency to the molecules. In these compounds, both potentials are generated on the one hand by the trimethoxyl groups and by the carbonyl on the indole on the other hand and are located at 10 and $8 \AA$ from each other in $\mathbf{1 6 b}$ and $\mathbf{1 7 b}$, respectively. We can thus assume that a hybrid 'cache-oreilles' system can be identified here as responsible for the anti-PAF activity. Nevertheless, the distance of $8 \AA$ observed in $\mathbf{1 7 b}$ is insufficient to enable a proper interaction of the molecule with the PAF-R but the activity of this molecule $\left(\mathrm{IC}_{50}\right.$ of $\left.11 \mu \mathrm{M}\right)$ suggests that a distance of $10-12 \AA$ must be reached in other conformation than the one used to realize its $3 \mathrm{D}$ electrostatic map.

In conclusion, the 3,4,5-trimethoxyphenyl moiety found in PMS 601, our reference compound, can be replaced by an indole without modifying either its anti-PAF or antiviral activity. The structure-activity relationships studied around this heterocycle led to compounds presenting a dual activity at the micromolar potency. The variations of the anti-PAF activity are in concordance with the PAF-receptor model previously proposed by our laboratory. The antiviral mechanism of this series of compounds is still unknown and the identification of their biological target is now a priority to greatly improve the antiviral activity of this piperazine series.

\section{Experimental}

\subsection{Materials and methods}

All materials were obtained from commercial suppliers and used without further purification. Thin-layer chromatography was performed on TLC plastic sheets of silica gel 60F254 (layer thickness $0.2 \mathrm{~mm}$ ) from Merck. Column chromatography purification was carried out with silica gel 60 (70-230 mesh ASTM, Merck). All melting points were determined on a digital melting point apparatus (Electrothermal) and are uncorrected. The structures of all compounds were confirmed by IR and NMR spectra. IR spectra were obtained with a ATI Mattson Genesis Series FTIR spectrometer, and ${ }^{1} \mathrm{H}$ NMR and ${ }^{13} \mathrm{C}$ NMR spectra were recorded in $\mathrm{CDCl}_{3}$ or in DMSO- $d_{6}$ on a BRUCKER AC 200 spectrometer using hexamethyldisiloxane (HMDS) as an internal standard. Chemical shifts are given in ppm and peak multiplicities are designated as follows: s, singlet; d, doublet; dd, doublet doublet; t, triplet; br s, broad singlet; m, multiplet; q, quadruplet; sex, sextuplet and sep, septuplet. Elemental analyses were obtained from the 'Service Régional de Microanalyse' (Université Pierre et Marie
Curie, Paris, France) and were within $0.4 \%$ of theoretical values.

3.1.1. General procedure for the synthesis of $3 a-j$ and 4a-f. To a solution of the amine $\mathbf{1}$ or $\mathbf{2}$ in $\mathrm{CH}_{2} \mathrm{Cl}_{2}$ were added the carboxylic acid ( 1 or 1.2 equiv), the $N, N^{\prime}$-dicyclohexylcarbodiimide, DCC (1 or 1.2 equiv) or $N, N^{\prime}$ diisopropylcarbodiimide, DIC (1 or 1.2 equiv) and, with or without 1-hydroxybenzotriazole, HOBT (1 or 1.2 equiv). The mixture was refluxed until the reaction was complete (TLC monitored). After cooling at room temperature and filtration, the mixture was washed with $\mathrm{HCl} 1 \mathrm{~N}$ and water, then with saturated $\mathrm{NaHCO}_{3}$ and twice with water. The organic layer was dried $\left(\mathrm{MgSO}_{4}\right)$ and concentrated in vacuo. The residue was chromatographed on a silica gel column as indicated.

3.1.2. $2-(\mathrm{N}, \mathrm{N}$-Diethylaminocarbonyloxymethyl)-1-(1Hindole-2-ylcarbonyl)-4-(3,4,5-trimethoxybenzoyl)piperazine (3a). Compound 3a was prepared using $1 H$-indole2-carboxylic acid (234 mg), DCC (301 mg), HOBT $(197 \mathrm{mg})$ and 1 (500 mg, $1.22 \mathrm{mmol})$, and obtained as a white solid $(600 \mathrm{mg}, 89 \%)$ after a silica gel column chromatography using $\mathrm{MeOH} / \mathrm{CH}_{2} \mathrm{Cl}_{2}(1: 99$, v/v) as eluent: $R_{\mathrm{f}} 0.36\left(\mathrm{MeOH} / \mathrm{CH}_{2} \mathrm{Cl}_{2}, 5: 95, \mathrm{v} / \mathrm{v}\right) ; \mathrm{mp} 148^{\circ} \mathrm{C}$; IR $\left(v \mathrm{~cm}^{-1}\right) 3380(\mathrm{NH}), 1697(\mathrm{C}=\mathrm{O}$ carbamate), 1636 $(\mathrm{C}=\mathrm{O}$ amide $), 1585(\mathrm{ArC}=\mathrm{C}) ;{ }^{1} \mathrm{H}$ NMR $\left(\mathrm{CDCl}_{3}, \delta\right)$ : 10.02 (br s, $1 \mathrm{H}, \mathrm{NH}), 7.55$ (d, $1 \mathrm{H}, J=8 \mathrm{~Hz}, \operatorname{ArH})$, 7.35 (d, $1 \mathrm{H}, J=8 \mathrm{~Hz}, \operatorname{ArH}), 7.22(\mathrm{~m}, 1 \mathrm{H}, \operatorname{ArH}), 7.08$ (m, 1H, ArH), 6.80 (s, 1H, ArH), 6.60 (s, 2H, ArH), $5.30-4.90\left(\mathrm{~m}, 1 \mathrm{H}, \quad \mathrm{CH}_{2} \mathrm{OC}=\mathrm{O}\right), 4.80-4.30(\mathrm{~m}, 3 \mathrm{H}$, $\mathrm{CH}_{2} \mathrm{OC}=\mathrm{O}$ and piperazine $), 4.20-4.00(\mathrm{~m}, 2 \mathrm{H}$, piperazine), $3.79\left(\mathrm{~s}, 9 \mathrm{H}, \mathrm{OCH}_{3}\right), 3.75-2.60\left(\mathrm{~m}, 7 \mathrm{H}, \mathrm{NCH}_{2} \mathrm{CH}_{3}\right.$ and piperazine), $1.01,0.98\left(2 \mathrm{~m}, 6 \mathrm{H}, \mathrm{NCH}_{2} \mathrm{CH}_{3}\right) ;{ }^{13} \mathrm{C}$ NMR $\left(\mathrm{CDCl}_{3}, \delta\right): 170.76,163.34(\mathrm{C}=\mathrm{O}$ amide $), 154.97$ $(\mathrm{C}=\mathrm{O}$ carbamate $), 153.30,139.33,135.95,130.03$, $128.45,127.13,124.45,121.79,120.46,111.74,105.82$, 104.23 $(\mathrm{ArC}=\mathrm{C}), 60.84\left(\mathrm{CH}_{2} \mathrm{O}\right), 60.72,56.16\left(\mathrm{CH}_{3} \mathrm{O}\right)$, 41.74, $41.00\left(\mathrm{NCH}_{2} \mathrm{CH}_{3}\right), 13.64,13.14\left(\mathrm{NCH}_{2} \mathrm{CH}_{3}\right)$. Anal. $\left(\mathrm{C}_{29} \mathrm{H}_{36} \mathrm{O}_{7} \mathrm{~N}_{4} \cdot 0.33 \mathrm{H}_{2} \mathrm{O}\right) \mathrm{C}, \mathrm{H}, \mathrm{N}$.

3.1.3. 2-(N,N-Diethylaminocarbonyloxymethyl)-1-(1 Hindole-3-ylcarbonyl)-4-(3,4,5-trimethoxybenzoyl)piperazine (3b). Compound $\mathbf{3 b}$ was prepared using $1 H$-indole3-carboxylic acid (197 mg), DCC (251 mg), HOBT $(197 \mathrm{mg}), 1(500 \mathrm{mg}, 1.22 \mathrm{mmol})$ and obtained as a white solid $(560 \mathrm{mg}, 83 \%)$ after a silica gel column chromatography using $\mathrm{MeOH} / \mathrm{CH}_{2} \mathrm{Cl}_{2}\left(1: 99\right.$, v/v) as eluent: $R_{\mathrm{f}} 0.38$ $\left(\mathrm{MeOH} / \mathrm{CH}_{2} \mathrm{Cl}_{2}, 5: 95, \mathrm{v} / \mathrm{v}\right) ; \mathrm{mp} 174{ }^{\circ} \mathrm{C}$; IR $\left(v \mathrm{~cm}^{-1}\right)$ $3192(\mathrm{NH}), 1698(\mathrm{C}=\mathrm{O}$ carbamate $), 1618,1604(\mathrm{C}=\mathrm{O}$ amide), $1582(\mathrm{ArC}=\mathrm{C}) ;{ }^{1} \mathrm{H}$ NMR $\left(\mathrm{CDCl}_{3}, \delta\right): 9.32(\mathrm{br}$ $\mathrm{s}, 1 \mathrm{H}, \mathrm{NH}), 7.60(\mathrm{~m}, 1 \mathrm{H}, \operatorname{ArH}), 7.45(\mathrm{~s}, 1 \mathrm{H}, \operatorname{ArH})$, $7.32(\mathrm{~m}, 1 \mathrm{H}, \operatorname{ArH}), 7.10(\mathrm{~m}, 2 \mathrm{H}, \operatorname{ArH}), 6.58(\mathrm{~s}, 2 \mathrm{H}$, $\mathrm{ArH}), \quad 4.94\left(\mathrm{~m}, 1 \mathrm{H}, \quad \mathrm{CH}_{2} \mathrm{OC}=\mathrm{O}\right), 4.44(\mathrm{~m}, 2 \mathrm{H}$, $\mathrm{CH}_{2} \mathrm{OC}=\mathrm{O}$ and piperazine $), 4.25-4.00(\mathrm{~m}, 2 \mathrm{H}$, piperazine $), 3.78\left(\mathrm{~s}, 9 \mathrm{H}, \mathrm{OCH}_{3}\right), \quad 3.71-2.90(\mathrm{~m}, 10 \mathrm{H}$, $\mathrm{NCH}_{2} \mathrm{CH}_{3}$ and piperazine), $1.02-0.81$ (m, $6 \mathrm{H}$, $\left.\mathrm{NCH}_{2} \mathrm{CH}_{3}\right) ;{ }^{13} \mathrm{C}$ NMR $\left(\mathrm{CDCl}_{3}, \delta\right): 170.97,167.84$ $(\mathrm{C}=\mathrm{O}$ amide $), \quad 155.20 \quad(\mathrm{C}=\mathrm{O}$ carbamate $), 153.25$, $139.24,135.78,130.05,127.77,125.28,122.57,120.96$, $119.87, \quad 111.82, \quad 109.68, \quad 104.20 \quad(\mathrm{ArC}=\mathrm{C}), \quad 60.93$ $\left(\mathrm{CH}_{2} \mathrm{O}\right), 60.72,56.13\left(\mathrm{CH}_{3} \mathrm{O}\right), 41.74,41.08\left(\mathrm{NCH}_{2} \mathrm{CH}_{3}\right)$, 13.65, $13.11\left(\mathrm{NCH}_{2} \mathrm{CH}_{3}\right)$. Anal. $\left(\mathrm{C}_{29} \mathrm{H}_{36} \mathrm{O}_{7} \mathrm{~N}_{4}\right) \mathrm{C}, \mathrm{H}, \mathrm{N}$. 
3.1.4. 1-(Benzothiophene-2-ylcarbonyl)-2-( $N, N$-diethylaminocarbonyloxymethyl)-4-(3,4,5-trimethoxybenzoyl)piperazine (3c). Compound $\mathbf{3 c}$ was prepared using benzothiophene-2-carboxylic acid (261 mg), DCC (301 mg), HOBT (197 mg), 1 (500 mg, $1.22 \mathrm{mmol})$ and obtained as a white solid (400 mg, 57\%) after a silica gel column chromatography using $\mathrm{MeOH} /$ $\mathrm{CH}_{2} \mathrm{Cl}_{2}(1: 99, \mathrm{v} / \mathrm{v})$ as eluent: $R_{\mathrm{f}} 0.43\left(\mathrm{CH}_{2} \mathrm{Cl}_{2} / \mathrm{MeOH}\right.$, 95:5, v/v); mp $105^{\circ} \mathrm{C}$; IR $\left(v \mathrm{~cm}^{-1}\right) 3379(\mathrm{NH}), 1698$ ( $\mathrm{C}=\mathrm{O} \quad$ carbamate), $1634 \quad(\mathrm{C}=\mathrm{O} \quad$ amide $), 1585$ $(\mathrm{ArC}=\mathrm{C}) ;{ }^{1} \mathrm{H} \mathrm{NMR}\left(\mathrm{CDCl}_{3}, \delta\right): 7.75(\mathrm{~m}, 2 \mathrm{H}, \mathrm{ArH})$, $7.46(\mathrm{~s}, 1 \mathrm{H}, \mathrm{ArH}), 7.35(\mathrm{~m}, 2 \mathrm{H}, \mathrm{ArH}), 6.58(\mathrm{~s}, 2 \mathrm{H}$, $\mathrm{ArH}), 4.50\left(\mathrm{~m}, 1 \mathrm{H}, \mathrm{CH}_{2} \mathrm{OC}=\mathrm{O}\right), 4.60-4.10(\mathrm{~m}, 5 \mathrm{H}$, $\mathrm{CH}_{2} \mathrm{OC}=\mathrm{O}$ and piperazine), 3.81 (s, 9H, $\left.\mathrm{OCH}_{3}\right)$, 3.70-2.60 (m, 7H, $\mathrm{NCH}_{2} \mathrm{CH}_{3}$ and piperazine), 0.97 $\left(\mathrm{m}, 6 \mathrm{H}, \mathrm{NCH}_{2} \mathrm{CH}_{3}\right) ;{ }^{13} \mathrm{C} \mathrm{NMR}\left(\mathrm{CDCl}_{3}, \delta\right): 170.78$, 164.57 ( $\mathrm{C}=\mathrm{O}$ amide), $154.99 \quad(\mathrm{C}=\mathrm{O}$ carbamate), $153.34,140.17,139.39,138.41,135.9,130.03,125.99$, 125.67, 124.86, 124.65, 122.27, 104.23 (ArC $=\mathrm{C})$, $60.76 \quad\left(\mathrm{CH}_{2} \mathrm{OC}=\mathrm{O}\right), \quad 60.69, \quad 56.21 \quad\left(\mathrm{CH}_{3} \mathrm{O}\right), 41.81$, $41.13\left(\mathrm{NCH}_{2} \mathrm{CH}_{3}\right), 13.83,13.25\left(\mathrm{NCH}_{2} \mathrm{CH}_{3}\right)$. Anal. $\left(\mathrm{C}_{29} \mathrm{H}_{35} \mathrm{O}_{7} \mathrm{~N}_{3} \mathrm{~S}\right) \mathrm{C}, \mathrm{H}, \mathrm{N}$.

3.1.5. 1-(Pyrrole-2-ylcarbonyl)-2-(N,N-diethylaminocarbonyloxymethyl)-4-(3,4,5-trimethoxybenzoyl)piperazine (3d). Compound 3d was prepared using pyrrole-2-carboxylic acid (325 mg), DCC (482 mg), 1 (800 mg, $1.95 \mathrm{mmol}$ ) and obtained as a white solid (450 mg, $52 \%$ ) after a silica gel column chromatography using $\mathrm{MeOH} / \mathrm{CH}_{2} \mathrm{Cl}_{2}\left(1: 99\right.$, v/v) as eluent: $R_{\mathrm{f}} 0.2\left(\mathrm{CH}_{2} \mathrm{Cl}_{2} /\right.$ $\mathrm{MeOH}, 95: 5, \mathrm{v} / \mathrm{v}) ; \operatorname{mp~} 86^{\circ} \mathrm{C}$; IR $\left(v \mathrm{~cm}^{-1}\right) 3293(\mathrm{NH})$, 1694 ( $\mathrm{C}=\mathrm{O}$ carbamate), 1633 ( $\mathrm{C}=\mathrm{O}$ amide), 1585 $(\mathrm{ArC}=\mathrm{C}) ;{ }^{1} \mathrm{H} \mathrm{NMR}\left(\mathrm{CDCl}_{3}, \delta\right): 10.07$ (br s, $\left.1 \mathrm{H}, \mathrm{NH}\right)$, $6.87(\mathrm{~s}, 1 \mathrm{H}, \mathrm{ArH}), 6.87-6.55(\mathrm{~m}, 3 \mathrm{H}, \mathrm{ArH}), 6.21(\mathrm{~s}$, $1 \mathrm{H}, \mathrm{ArH}), 5.20-3.98\left(\mathrm{~m}, 6 \mathrm{H}, \mathrm{CH}_{2} \mathrm{OC}=\mathrm{O}\right.$ and piperazine), $3.83\left(\mathrm{~s}, 9 \mathrm{H}, \mathrm{OCH}_{3}\right), 3.83-2.50\left(\mathrm{~m}, 7 \mathrm{H}, \mathrm{NCH}_{2} \mathrm{CH}_{3}\right.$ and piperazine $), 1.00,0.83\left(2 \mathrm{~m}, 6 \mathrm{H}, \mathrm{NCH}_{2} \mathrm{CH}_{3}\right) ;{ }^{13} \mathrm{C}$ NMR $\left(\mathrm{CDCl}_{3}, \delta\right): 170.75,162.44(\mathrm{C}=\mathrm{O}$ amide $), 154.93$ $(\mathrm{C}=\mathrm{O}$ carbamate $), 153.28,139.29, \quad 130.15123 .73$, 121.61, 112.69, 109.54, 104.24 (ArC=C), 60.80 $\left(\mathrm{CH}_{2} \mathrm{OC}=\mathrm{O}\right), \quad 60.72, \quad 56.17 \quad\left(\mathrm{CH}_{3} \mathrm{O}\right), \quad 41.70,40.93$ $\left(\mathrm{NCH}_{2} \mathrm{CH}_{3}\right), \quad 13.59, \quad 13.18 \quad\left(\mathrm{NCH}_{2} \mathrm{CH}_{3}\right)$. Anal. $\left(\mathrm{C}_{25} \mathrm{H}_{34} \mathrm{O}_{7} \mathrm{~N}_{4} \cdot 0.5 \mathrm{H}_{2} \mathrm{O}\right) \mathrm{C}, \mathrm{H}, \mathrm{N}$.

3.1.6. 1-(5-Chloro-1 $H$-indole-2-ylcarbonyl)-2-( $N, N$-diethylaminocarbonyloxymethyl)-4-(3,4,5-trimethoxybenzoyl)piperazine (3e). Compound $\mathbf{3 e}$ was prepared using 5-chloro- $1 H$-indole-2-carboxylic acid $(555 \mathrm{mg})$, DCC (602 mg), 1 (1 g, $2.44 \mathrm{mmol})$ and obtained as a white solid $(1 \mathrm{~g}, 66 \%)$ after a silica gel column chromatography using $\mathrm{MeOH} / \mathrm{CH}_{2} \mathrm{Cl}_{2}\left(1: 99\right.$, v/v) as eluent: $R_{\mathrm{f}} 0.30$ $\left(\mathrm{CH}_{2} \mathrm{Cl}_{2} / \mathrm{MeOH}, 95: 5, \mathrm{v} / \mathrm{v}\right) ; \mathrm{mp} 168^{\circ} \mathrm{C}$; IR $\left(v \mathrm{~cm}^{-1}\right)$ 3263 (NH), 1704 ( $\mathrm{C}=\mathrm{O}$ carbamate), 1634 ( $\mathrm{C}=\mathrm{O}$ amide), $1595(\mathrm{ArC}=\mathrm{C}) ;{ }^{1} \mathrm{H} \mathrm{NMR}\left(\mathrm{CDCl}_{3}, \delta\right): 9.83$ (br s, $1 \mathrm{H}$, $\mathrm{NH}), 7.58(\mathrm{~s}, 1 \mathrm{H}, \mathrm{ArH}), 7.36(\mathrm{~d}, 1 \mathrm{H}, J=8.7 \mathrm{~Hz}$, $\mathrm{ArH}), 7.22$ (d, $1 \mathrm{H}, J=8.7 \mathrm{~Hz}, \mathrm{ArH}), 6.80(\mathrm{~s}, 1 \mathrm{H}$, $\mathrm{ArH}), 6.65$ (s, 2H, ArH), 5.10-4.00 (m, 6H, $\mathrm{CH}_{2} \mathrm{OC}=\mathrm{O}$ and piperazine $), 3.86\left(\mathrm{~s}, 9 \mathrm{H}, \mathrm{OCH}_{3}\right), 3.50-2.80(\mathrm{~m}, 7 \mathrm{H}$, $\mathrm{NCH}_{2} \mathrm{CH}_{3}$ and piperazine), 1.07, $0.86(2 \mathrm{~m}, 6 \mathrm{H}$, $\left.\mathrm{NCH}_{2} \mathrm{CH}_{3}\right) ;{ }^{13} \mathrm{C} \mathrm{NMR}\left(\mathrm{CDCl}_{3}, \delta\right): 170.77,162.80(\mathrm{C}=\mathrm{O}$ amide), 155.00 ( $\mathrm{C}=\mathrm{O}$ carbamate), 153.32, 139.43, 134.13, $129.90,129.76,128.09,126.09,125.01,120.98,112.78$, 105.31, 104.26 (ArC=C), $60.84 \quad\left(\mathrm{CH}_{2} \mathrm{OC}=\mathrm{O}\right), 60.73$,
$56.18\left(\mathrm{CH}_{3} \mathrm{O}\right), 41.77,40.99\left(\mathrm{NCH}_{2} \mathrm{CH}_{3}\right), 13.63,13.14$ $\left(\mathrm{NCH}_{2} \mathrm{CH}_{3}\right)$. Anal. $\left(\mathrm{C}_{29} \mathrm{H}_{35} \mathrm{O}_{7} \mathrm{~N}_{4} \mathrm{Cl}\right) \mathrm{C}, \mathrm{H}, \mathrm{N}$.

3.1.7. 2-(N,N-Diethylaminocarbonyloxymethyl)-1-(5methoxy-1 $H$-indole-2-ylcarbonyl)-4-(3,4,5-trimethoxybenzoyl)piperazine (3f). Compound $\mathbf{3 f}$ was prepared using 5-methoxy-1H-indole-2-carboxylic acid (514 mg), DCC (510 mg), 1 (850 mg, $2.07 \mathrm{mmol})$ and obtained as a white solid $(840 \mathrm{mg}, 70 \%)$ after a silica gel column chromatography using $\mathrm{MeOH} / \mathrm{CH}_{2} \mathrm{Cl}_{2}(1: 99$, v/v) as eluent: $R_{\mathrm{f}} 0.26\left(\mathrm{CH}_{2} \mathrm{Cl}_{2} / \mathrm{MeOH}, 95: 5\right.$, v/v); mp $174{ }^{\circ} \mathrm{C}$; IR $\left(v \mathrm{~cm}^{-1}\right) 3268(\mathrm{NH}), 1700(\mathrm{C}=\mathrm{O}$ carbamate $), 1635$ $(\mathrm{C}=\mathrm{O}$ amide $), 1596(\mathrm{ArC}=\mathrm{C}) ;{ }^{1} \mathrm{H} \mathrm{NMR}\left(\mathrm{CDCl}_{3}, \delta\right)$ : $8.72(\mathrm{~s}, 1 \mathrm{H}, \mathrm{NH}), 7.32(\mathrm{~d}, 1 \mathrm{H}, J=8.8 \mathrm{~Hz}, \mathrm{ArH}), 7.01$ (s, 1H, ArH), 6.95 (d, 1H, J=8.8 Hz, ArH), 6.78 (s, $1 \mathrm{H}, \mathrm{ArH}), 6.65$ (s, 2H, ArH), 5.20-3.96 (m, 6H, $\mathrm{CH}_{2} \mathrm{OC}=\mathrm{O}$ and piperazine $), 3.86,3.81(2 \mathrm{~s}, 12 \mathrm{H}$, $\left.\mathrm{OCH}_{3}\right), 3.70-3.01\left(\mathrm{~m}, 7 \mathrm{H}, \mathrm{NCH}_{2} \mathrm{CH}_{3}\right.$ and piperazine), $1.00,0.85\left(2 \mathrm{~m}, 6 \mathrm{H}, \mathrm{NCH}_{2} \mathrm{CH}_{3}\right) ;{ }^{13} \mathrm{C} \mathrm{NMR}\left(\mathrm{CDCl}_{3}, \delta\right)$ : $170.80,163.26(\mathrm{C}=\mathrm{O}$ amide $), 155.00(\mathrm{C}=\mathrm{O}$ carbamate $)$, $154.52,153.35,139.40,131.30,130.07,128.90,127.55$, 115.95, 112.64, 105.57, 104.29, 102.09 (ArC=C), 60.88 $\left(\mathrm{CH}_{2} \mathrm{OC}=\mathrm{O}\right), \quad 60.77, \quad 56.22 \quad\left(\mathrm{CH}_{3} \mathrm{O}\right), 55.58 \quad\left(\mathrm{CH}_{3} \mathrm{O}\right)$, 41.80, $41.07\left(\mathrm{NCH}_{2} \mathrm{CH}_{3}\right), 13.70,13.21\left(\mathrm{NCH}_{2} \mathrm{CH}_{3}\right)$. Anal. $\left(\mathrm{C}_{30} \mathrm{H}_{38} \mathrm{O}_{8} \mathrm{~N}_{4}\right) \mathrm{C}, \mathrm{H}, \mathrm{N}$.

3.1.8. 1-(Benzofurane-2-ylcarbonyl)-2-( $N, N$-diethylaminocarbonyloxymethyl)-4-(3,4,5-trimethoxybenzoyl)piperazine (3g). Compound $\mathbf{3 g}$ was prepared using benzofuran-2-carboxylic acid (197 mg), DCC (251 mg), HOBT (197 mg), 1 (500 mg, $1.22 \mathrm{mmol}$ ) and obtained as a white solid (450 mg, 67\%) after a silica gel column chromatography using $\mathrm{MeOH} / \mathrm{CH}_{2} \mathrm{Cl}_{2}(1: 99$, v/v) as eluent: $R_{\mathrm{f}} 0.27\left(\mathrm{CH}_{2} \mathrm{Cl}_{2} / \mathrm{MeOH}, 95: 5, \mathrm{v} / \mathrm{v}\right) ; \mathrm{mp} 102{ }^{\circ} \mathrm{C}$; IR $\left(v \mathrm{~cm}^{-1}\right) 2999(\mathrm{ArCH}), 1702(\mathrm{C}=\mathrm{O}$ carbamate $), 1618$ $\left(\mathrm{C}=\mathrm{O}\right.$ amide), $1583(\mathrm{ArC}=\mathrm{C}) ;{ }^{1} \mathrm{H} \mathrm{NMR}\left(\mathrm{CDCl}_{3}, \delta\right)$ : $7.58(\mathrm{~d}, 1 \mathrm{H}, J=8 \mathrm{~Hz}, \operatorname{ArH}), 7.44(\mathrm{~d}, 1 \mathrm{H}, J=8 \mathrm{~Hz}$, $\mathrm{ArH}), 7.43,7.19(\mathrm{~m}, 3 \mathrm{H}, \mathrm{ArH}), 6.60$ (s, 2H, ArH), $5.06\left(\mathrm{~m}, \quad 1 \mathrm{H}, \quad \mathrm{CH}_{2} \mathrm{OC}=\mathrm{O}\right), 4.80,4.00 \quad(2 \mathrm{~m}, 4 \mathrm{H}$, $\mathrm{CH}_{2} \mathrm{OC}=\mathrm{O}$ and piperazine $), 3.82\left(\mathrm{~s}, 9 \mathrm{H}, \mathrm{OCH}_{3}\right), 3.59-$ $2.80\left(\mathrm{~m}, 8 \mathrm{H}, \mathrm{NCH}_{2} \mathrm{CH}_{3}\right.$ and piperazine), 1.10, 0.91 $\left(2 \mathrm{~m}, 6 \mathrm{H}, \mathrm{NCH}_{2} \mathrm{CH}_{3}\right) ;{ }^{13} \mathrm{C} \mathrm{NMR}\left(\mathrm{CDCl}_{3}, \delta\right): 170.74$, $160.42(\mathrm{C}=\mathrm{O}$ amide $), \quad 154.85 \quad(\mathrm{C}=\mathrm{O}$ carbamate $)$, $154.54,153.33,148.29,139.36,130.06,126.60,125.99$, 125.67, 124.86, 124.65, 122.27, 104.23 (ArC=C), 60.82 $\left(\mathrm{CH}_{2} \mathrm{OC}=\mathrm{O}\right), \quad 60.75, \quad 56.20 \quad\left(\mathrm{CH}_{3} \mathrm{O}\right), \quad 41.70, \quad 41.00$ $\left(\mathrm{NCH}_{2} \mathrm{CH}_{3}\right), \quad 13.69, \quad 13.18 \quad\left(\mathrm{NCH}_{2} \mathrm{CH}_{3}\right)$. Anal. $\left(\mathrm{C}_{29} \mathrm{H}_{35} \mathrm{O}_{8} \mathrm{~N}_{3}\right) \mathrm{C}, \mathrm{H}, \mathrm{N}$.

3.1.9. 2-( $N, N$-Diethylaminocarbonyloxymethyl)-1-(quinoline-2-ylcarbonyl)-4-(3,4,5- trimethoxybenzoyl)piperazine (3h). Compound $3 \mathbf{h}$ was prepared using quinoline-2-carboxylic acid (463 mg), DCC (431 mg), 1 (730 mg, $1.78 \mathrm{mmol}$ ) and obtained as a white solid (760 mg, $76 \%$ ) after a silica gel column chromatography using $\mathrm{MeOH} / \mathrm{CH}_{2} \mathrm{Cl}_{2}\left(1: 99\right.$, v/v) as eluent: $R_{\mathrm{f}} 0.32\left(\mathrm{CH}_{2} \mathrm{Cl}_{2} /\right.$ $\mathrm{MeOH}, 95: 5, \mathrm{v} / \mathrm{v}) ; \operatorname{mp~} 72^{\circ} \mathrm{C}$; IR $\left(v \mathrm{~cm}^{-1}\right) 3270(\mathrm{NH})$, $1705(\mathrm{C}=\mathrm{O}$ carbamate), 1635 ( $\mathrm{C}=\mathrm{O}$ amide), 1597 $(\mathrm{ArC}=\mathrm{C}) ;{ }^{1} \mathrm{H} \quad \mathrm{NMR} \quad\left(\mathrm{CDCl}_{3}, \delta\right): 8.27 \quad(\mathrm{~d}, \quad 1 \mathrm{H}$, $J=8.49 \mathrm{~Hz}, \mathrm{ArH}), 8.06(\mathrm{~d}, 1 \mathrm{H}, J=8.42 \mathrm{~Hz}, \mathrm{ArH})$, $7.86(\mathrm{~d}, 1 \mathrm{H}, J=8.2 \mathrm{~Hz}, \mathrm{ArH}), 7.78-7.56(\mathrm{~m}, 3 \mathrm{H}$, $\mathrm{ArH}), 6.63$ (s, 2H, ArH), 5.40-4.00 (m, 6H, $\mathrm{CH}_{2} \mathrm{OC}=\mathrm{O}$ and piperazine $), 3.86\left(\mathrm{~s}, 9 \mathrm{H}, \mathrm{OCH}_{3}\right), 3.71-2.70(\mathrm{~m}, 7 \mathrm{H}$, 
$\mathrm{NCH}_{2} \mathrm{CH}_{3}$ and piperazine), 1.10, $1.00(2 \mathrm{~m}, 6 \mathrm{H}$, $\left.\mathrm{NCH}_{2} \mathrm{CH}_{3}\right) ;{ }^{13} \mathrm{C}$ NMR $\left(\mathrm{CDCl}_{3}, \delta\right): 170.90,168.17$ $(\mathrm{C}=\mathrm{O}$ amide $), \quad 153.36 \quad(\mathrm{C}=\mathrm{O}$ carbamate $), 146.14$, $137.00,130.32,128.04,137.24,130.17,129.74,127.88$, $127.59, \quad 121.22, \quad 120.65, \quad 104.21 \quad(\mathrm{ArC}=\mathrm{C}) \quad 61.38$ $\left(\mathrm{CH}_{2} \mathrm{OC}=\mathrm{O}\right), \quad 60.80, \quad 56.24 \quad\left(\mathrm{CH}_{3} \mathrm{O}\right), \quad 41.81, \quad 41.15$ $\left(\mathrm{NCH}_{2} \mathrm{CH}_{3}\right), \quad 13.88, \quad 13.36 \quad\left(\mathrm{NCH}_{2} \mathrm{CH}_{3}\right)$. Anal. $\left(\mathrm{C}_{30} \mathrm{H}_{36} \mathrm{O}_{7} \mathrm{~N}_{4}\right) \mathrm{C}, \mathrm{H}, \mathrm{N}$.

3.1.10. 2-(N,N-Diethylaminocarbonyloxymethyl)-1-(1 Hindole-3-ylacetyl)-4-(3,4,5-trimethoxybenzoyl)piperazine (3i). Compound 3i was prepared using $1 H$-indole-3acetic acid $(257 \mathrm{mg})$, DCC (301 mg), HOBT (197 mg), $1(500 \mathrm{mg}, 1.22 \mathrm{mmol})$ and obtained as a white solid (520 mg, 75\%) after a silica gel column chromatography using $\mathrm{MeOH} / \mathrm{CH}_{2} \mathrm{Cl}_{2}(2: 98, \mathrm{v} / \mathrm{v})$ as eluent: $R_{\mathrm{f}} 0.16$ $\left(\mathrm{CH}_{2} \mathrm{Cl}_{2} / \mathrm{MeOH}, 95: 5, \mathrm{v} / \mathrm{v}\right) ; \mathrm{mp} 97^{\circ} \mathrm{C}$; IR $\left(v \mathrm{~cm}^{-1}\right)$ $3192(\mathrm{NH}), 1685(\mathrm{C}=\mathrm{O}$ carbamate $), 1645(\mathrm{C}=\mathrm{O}$ amide $)$, $1583(\mathrm{ArC}=\mathrm{C}) ;{ }^{1} \mathrm{H}$ NMR $\left(\mathrm{CDCl}_{3}, \delta\right): 8.48(\mathrm{br} \mathrm{s}, 1 \mathrm{H}$, $\mathrm{NH}), 7.55(\mathrm{~d}, 1 \mathrm{H}, J=8 \mathrm{~Hz}, \operatorname{ArH}), 7.29-7.00(\mathrm{~m}, 4 \mathrm{H}$, ArH), 6.49 (s, 2H, ArH), 5.10, $4.10\left(2 \mathrm{~m}, 3 \mathrm{H}, \mathrm{CH}_{2} \mathrm{OC}=\mathrm{O}\right.$ and piperazine), $4.05-3.86\left(\mathrm{~m}, 5 \mathrm{H}, \mathrm{CH}_{2} \mathrm{CO}\right.$ and piperazine), $3.76\left(\mathrm{~s}, 9 \mathrm{H}, \mathrm{OCH}_{3}\right), 3.60-2.50\left(\mathrm{~m}, 7 \mathrm{H}, \mathrm{NCH}_{2} \mathrm{CH}_{3}\right.$ and piperazine), $1.01\left(\mathrm{~m}, 6 \mathrm{H}, \mathrm{NCH}_{2} \mathrm{CH}_{3}\right) ;{ }^{13} \mathrm{C} \mathrm{NMR}$ $\left(\mathrm{CDCl}_{3}, \delta\right): 171.25,170.79 \quad(\mathrm{C}=\mathrm{O}$ amide $), 155.02$ $(\mathrm{C}=\mathrm{O}$ carbamate $), 153.27,139.27,136.18,130.11$, $126.67,122.15,119.55,118.54,111.28,108.61,104.18$ $(\mathrm{ArC}=\mathrm{C}), \quad 61.03 \quad\left(\mathrm{CH}_{2} \mathrm{C}=\mathrm{O}\right), \quad 60.74 \quad\left(\mathrm{CH}_{2} \mathrm{OC}=\mathrm{O}\right)$, 60.51, 56.16 $\left(\mathrm{CH}_{3} \mathrm{O}\right), 41.88,41.23\left(\mathrm{NCH}_{2} \mathrm{CH}_{3}\right), 13.88$, $13.32\left(\mathrm{NCH}_{2} \mathrm{CH}_{3}\right)$. Anal. $\left(\mathrm{C}_{30} \mathrm{H}_{38} \mathrm{O}_{7} \mathrm{~N}_{4}\right) \mathrm{C}, \mathrm{H}, \mathrm{N}$.

3.1.11. $2-(N, N$-Diethylaminocarbonyloxymethyl)-1- $(1 H-$ indole-3-ylpropionyl)-4-(3,4,5-trimethoxybenzoyl)piperazine (3j). Compound $3 \mathbf{j}$ was prepared using ( $1 \mathrm{H}$-indole3-propionic acid $(231 \mathrm{mg})$, DCC $(251 \mathrm{mg})$, HOBT $(197 \mathrm{mg}), 1(500 \mathrm{mg}, 1.22 \mathrm{mmol})$ and obtained as a white solid $(600 \mathrm{mg}, 85 \%)$ after a silica gel column chromatography using $\mathrm{MeOH} / \mathrm{CH}_{2} \mathrm{Cl}_{2}(2: 98, \mathrm{v} / \mathrm{v})$ as eluent: $R_{\mathrm{f}} 0.2$ $\left(\mathrm{CH}_{2} \mathrm{Cl}_{2} / \mathrm{MeOH}, 95: 5, \mathrm{v} / \mathrm{v}\right) ; \mathrm{mp} 100{ }^{\circ} \mathrm{C}$; IR $\left(v \mathrm{~cm}^{-1}\right)$ $3310(\mathrm{NH}), 1696(\mathrm{C}=\mathrm{O}$ carbamate $), 1633(\mathrm{C}=\mathrm{O}$ amide $)$, $1582(\mathrm{ArC}=\mathrm{C}) ;{ }^{1} \mathrm{H}$ NMR $\left(\mathrm{CDCl}_{3}, \delta\right): 8.08(\mathrm{~s}, 1 \mathrm{H}, \mathrm{NH})$, 7.53 (s, $1 \mathrm{H}, \operatorname{ArH}), 7.29$ (d, $1 \mathrm{H}, J=7.8 \mathrm{~Hz}, \operatorname{ArH}), 7.25$, $6.90(\mathrm{~m}, 3 \mathrm{H}, \mathrm{ArH}), 6.49(\mathrm{~s}, 2 \mathrm{H}, \mathrm{ArH}), 5.20-4.30(\mathrm{~m}$, $\left.1 \mathrm{H}, \mathrm{CH}_{2} \mathrm{OC}=\mathrm{O}\right), 4.25-3.80\left(\mathrm{~m}, 4 \mathrm{H}, \mathrm{CH}_{2} \mathrm{OC}=\mathrm{O}\right.$ and piperazine $), 3.69\left(\mathrm{~s}, 9 \mathrm{H}, \mathrm{OCH}_{3}\right), 3.59-3.40(\mathrm{~m}, 1 \mathrm{H}$, $\left.\mathrm{CH}_{2} \mathrm{CO}\right), 3.30,2.30\left(2 \mathrm{~m}, 11 \mathrm{H}, \mathrm{CH}_{2} \mathrm{CH}_{2} \mathrm{CO}, \mathrm{NCH}_{2} \mathrm{CH}_{3}\right.$ and piperazine), $1.02,0.81\left(2 \mathrm{~m}, 6 \mathrm{H}, \mathrm{NCH}_{2} \mathrm{CH}_{3}\right) ;{ }^{13} \mathrm{C}$ NMR $\left(\mathrm{CDCl}_{3}, \delta\right): 172.45,170.75(\mathrm{C}=\mathrm{O}$ amide $), 154.89$ $(\mathrm{C}=\mathrm{O}$ carbamate $), 153.30,139.36,136.23,130.17$, $127.18,121.99,121.77,119.32,118.66,1180.49$, 114.73, $111.24(\mathrm{ArC}=\mathrm{C}), \quad 60.80 \quad\left(\mathrm{CH}_{2} \mathrm{OC}=\mathrm{O}\right), \quad 60.55, \quad 56.21$ $\left(\mathrm{CH}_{3} \mathrm{O}\right), 41.83,41.10\left(\mathrm{NCH}_{2} \mathrm{CH}_{3}\right), 34.13\left(\mathrm{CH}_{2} \mathrm{CO}\right)$, $21.43\left(\mathrm{CH}_{2} \mathrm{CH}_{2} \mathrm{CO}\right), 13.83,13.31\left(\mathrm{NCH}_{2} \mathrm{CH}_{3}\right)$. Anal. $\left(\mathrm{C}_{31} \mathrm{H}_{40} \mathrm{O}_{7} \mathrm{~N}_{4}\right) \mathrm{C}, \mathrm{H}, \mathrm{N}$.

3.1.12. $2-(N, N$-Diethylaminocarbonyloxyméthyl)-4-(1Hindole-2-ylcarbonyl)-1-(3,4,5-trimethoxybenzoyl)piperazine (4a). Compound $\mathbf{4 a}$ was prepared using $1 \mathrm{H}$-indole2-carboxylic acid $(234 \mathrm{mg})$, DCC (301 mg), HOBT (197 mg), 2 (500 mg, $1.22 \mathrm{mmol}$ ) and obtained as a white solid (500 $\mathrm{mg}, 74 \%$ ) after a silica gel column chromatography using $\mathrm{CH}_{2} \mathrm{Cl}_{2}$ as eluent: $R_{\mathrm{f}} 0.43\left(\mathrm{CH}_{2} \mathrm{Cl}_{2} / \mathrm{MeOH}\right.$, 95:5, v/v); mp $159.6{ }^{\circ} \mathrm{C}$; IR $\left(v \mathrm{~cm}^{-1}\right) 3370(\mathrm{NH}), 1698$
$(\mathrm{C}=\mathrm{O}$ carbamate $), 1644(\mathrm{C}=\mathrm{O}$ amide $), 1585(\mathrm{ArC}=\mathrm{C})$; ${ }^{1} \mathrm{H}$ NMR $\left(\mathrm{CDCl}_{3}, \delta\right): 9.46(\mathrm{br} \mathrm{s}, 1 \mathrm{H}, \mathrm{NH}), 7.55(\mathrm{~d}$, $1 \mathrm{H}, J=8 \mathrm{~Hz}, \operatorname{ArH}), 7.35$ (d, $1 \mathrm{H}, J=8 \mathrm{~Hz}, \operatorname{ArH}), 7.21$ $(\mathrm{m}, 1 \mathrm{H}, \mathrm{ArH}), 7.06(\mathrm{~m}, 1 \mathrm{H}, \mathrm{ArH}), 6.71(\mathrm{~s}, 1 \mathrm{H}, \mathrm{ArH})$, $6.60(\mathrm{~s}, 2 \mathrm{H}, \mathrm{ArH}), 5.20-4.30\left(\mathrm{~m}, 5 \mathrm{H}, \mathrm{CH}_{2} \mathrm{OC}=\mathrm{O}\right.$ and piperazine), $4.25-40(\mathrm{~m}, 1 \mathrm{H}$, piperazine $), 3.80(\mathrm{~s}, 9 \mathrm{H}$, $\left.\mathrm{OCH}_{3}\right), 3.70,2.80\left(2 \mathrm{~m}, 7 \mathrm{H}, \mathrm{NCH}_{2} \mathrm{CH}_{3}\right.$ and piperazine $)$, $0.95\left(\mathrm{~m}, 6 \mathrm{H}, \mathrm{NCH}_{2} \mathrm{CH}_{3}\right) ;{ }^{13} \mathrm{C} \mathrm{NMR}\left(\mathrm{CDCl}_{3}, \delta\right): 170.72$, $163.44(\mathrm{C}=\mathrm{O}$ amide $), 155.16(\mathrm{C}=\mathrm{O}$ carbamate $), 153.37$, $139.53,135.84,130.32,128.37,127.25,124.59,121.81$, $120.66, \quad 111.81, \quad 105.33, \quad 104.40 \quad(\mathrm{ArC}=\mathrm{C}), \quad 61.12$ $\left(\mathrm{CH}_{2} \mathrm{OC}=\mathrm{O}\right), \quad 60.85, \quad 56.23 \quad\left(\mathrm{CH}_{3} \mathrm{O}\right), \quad 41.82,41.11$ $\left(\mathrm{NCH}_{2} \mathrm{CH}_{3}\right), \quad 13.89, \quad 13.32 \quad\left(\mathrm{NCH}_{2} \mathrm{CH}_{3}\right)$. Anal. $\left(\mathrm{C}_{29} \mathrm{H}_{36} \mathrm{O}_{7} \mathrm{~N}_{4}\right) \mathrm{C}, \mathrm{H}, \mathrm{N}$.

3.1.13. 2-(N,N-Diethylaminocarbonyloxymethyl)-4-(1 Hindole-3-ylcarbonyl)-1-(3,4,5-trimethoxybenzoyl)piperazine (4b). Compound $\mathbf{4 b}$ was prepared using $1 H$-indole3-carboxylic acid (197 mg), DCC (251 mg), HOBT $(197 \mathrm{mg}), 2(500 \mathrm{mg}, 1.22 \mathrm{mmol})$ and obtained as a white solid (580 mg, 86\%) after a silica gel column chromatography using $\mathrm{MeOH} / \mathrm{CH}_{2} \mathrm{Cl}_{2}(1: 99, \mathrm{v} / \mathrm{v})$ as eluent: $R_{\mathrm{f}} 0.42$ $\left(\mathrm{MeOH} / \mathrm{CH}_{2} \mathrm{Cl}_{2}, 5: 95, \mathrm{v} / \mathrm{v}\right) ; \mathrm{mp} 201^{\circ} \mathrm{C}$; IR $\left(v \mathrm{~cm}^{-1}\right)$ $3171(\mathrm{NH}), 1699(\mathrm{C}=\mathrm{O}$ carbamate $), 1629(\mathrm{C}=\mathrm{O}$ amide $)$, $1599(\mathrm{ArC}=\mathrm{C}) ;{ }^{1} \mathrm{H}$ NMR $\left(\mathrm{CDCl}_{3}, \delta\right): 9.35(\mathrm{br} \mathrm{s}, 1 \mathrm{H}$, NH), $7.58(\mathrm{~m}, 1 \mathrm{H}, \mathrm{ArH}), 7.32(\mathrm{~m}, 2 \mathrm{H}, \mathrm{ArH}), 7.20(\mathrm{~m}$, $2 \mathrm{H}, \operatorname{ArH}), 6.58(\mathrm{~s}, 2 \mathrm{H}, \operatorname{ArH}), 5.10-3.90(\mathrm{~m}, 5 \mathrm{H}$, $\mathrm{CH}_{2} \mathrm{OC}=\mathrm{O}$ and piperazine $), 3.78\left(\mathrm{~s}, 9 \mathrm{H}, \mathrm{OCH}_{3}\right), 3.69$ $2.87\left(\mathrm{~m}, 8 \mathrm{H}, \mathrm{NCH}_{2} \mathrm{CH}_{3}\right.$ and piperazine $), 0.97(\mathrm{~m}, 6 \mathrm{H}$, $\left.\mathrm{NCH}_{2} \mathrm{CH}_{3}\right) ;{ }^{13} \mathrm{C}$ NMR $\left(\mathrm{CDCl}_{3}, \delta\right): 170.71,167.55$ $(\mathrm{C}=\mathrm{O}$ amide $), \quad 155.30 \quad(\mathrm{C}=\mathrm{O}$ carbamate $), 153.20$, $139.44,135.69,130.50,127.58,125.12,122.92,121.33$, $120.11, \quad 111.85, \quad 110.52, \quad 104.42 \quad(\mathrm{ArC}=\mathrm{C}), \quad 61.29$ $\left(\mathrm{CH}_{2} \mathrm{O}\right), 60.87,56.24\left(\mathrm{CH}_{3} \mathrm{O}\right), 41.87,41.23\left(\mathrm{NCH}_{2} \mathrm{CH}_{3}\right)$, 13.92, $13.46\left(\mathrm{NCH}_{2} \mathrm{CH}_{3}\right)$. Anal. $\left(\mathrm{C}_{29} \mathrm{H}_{36} \mathrm{O}_{7} \mathrm{~N}_{4}\right) \mathrm{C}, \mathrm{H}, \mathrm{N}$.

3.1.14. 4-(Benzothiophene-2-ylcarbonyl)-2-( $N, N$-diethylaminocarbonyloxymethyl)-1-(3,4,5-trimethoxybenzoyl)piperazine $(\mathbf{4 c})$. Compound $\mathbf{4 c}$ was prepared using benzothiophene-2-carboxylic acid $(430.2 \mathrm{mg})$, DIC $(375.7 \mu \mathrm{L}), 2(830 \mathrm{mg}, 2.19 \mathrm{mmol})$ and obtained as a white solid $(980 \mathrm{mg}, 83 \%)$ after a silica gel column chromatography using $\mathrm{MeOH} / \mathrm{CH}_{2} \mathrm{Cl}_{2}$ (1:99, v/v) as eluent: $R_{\mathrm{f}} 0.38\left(\mathrm{CH}_{2} \mathrm{Cl}_{2} / \mathrm{MeOH}, 95: 5, \mathrm{v} / \mathrm{v}\right) ; \mathrm{mp} 75^{\circ} \mathrm{C}$; IR $\left(v \mathrm{~cm}^{-1}\right) 3379(\mathrm{NH}), 1698 \quad(\mathrm{C}=\mathrm{O}$ carbamate $)$, $1634(\mathrm{C}=\mathrm{O}$ amide $), 1585(\mathrm{ArC}=\mathrm{C}) ;{ }^{1} \mathrm{H}$ NMR $\left(\mathrm{CDCl}_{3}\right.$, $\delta): 7.82(\mathrm{~m}, 2 \mathrm{H}, \mathrm{ArH}), 7.49(\mathrm{~s}, 1 \mathrm{H}, \mathrm{ArH}), 7.38(\mathrm{~m}$, $2 \mathrm{H}, \quad \operatorname{ArH}), 6.62(\mathrm{~s}, 2 \mathrm{H}, \operatorname{ArH}), \quad 5.52(\mathrm{~m}, 1 \mathrm{H}$, $\left.\mathrm{CH}_{2} \mathrm{OC}=\mathrm{O}\right), 4.55-4.20\left(\mathrm{~m}, 3 \mathrm{H}, \mathrm{CH}_{2} \mathrm{OC}=\mathrm{O}\right.$ and piperazine), 4.00-3.40 (m, 2H, piperazine), $3.84(\mathrm{~s}, 9 \mathrm{H}$, $\left.\mathrm{OCH}_{3}\right), 3.70-2.80\left(\mathrm{~m}, 7 \mathrm{H}, \mathrm{NCH}_{2} \mathrm{CH}_{3}\right.$ and piperazine), $1.07,0.88\left(2 \mathrm{~m}, 6 \mathrm{H}, \mathrm{NCH}_{2} \mathrm{CH}_{3}\right) ;{ }^{13} \mathrm{C} \mathrm{NMR}\left(\mathrm{CDCl}_{3}\right.$, $\delta): 170.67,164.79(\mathrm{C}=\mathrm{O}$ amide $), 155.10(\mathrm{C}=\mathrm{O}$ carbamate), $153.31,140.14,138.40,135.34,130.20,126.00$, $125.76, \quad 124.90, \quad 124.65, \quad 122.32,104.29 \quad(\mathrm{ArC}=\mathrm{C})$, $60.96 \quad\left(\mathrm{CH}_{2} \mathrm{OC}=\mathrm{O}\right), \quad 60.82, \quad 56.18 \quad\left(\mathrm{CH}_{3} \mathrm{O}\right), \quad 41.82$, $41.06\left(\mathrm{NCH}_{2} \mathrm{CH}_{3}\right), 13.78,13.37\left(\mathrm{NCH}_{2} \mathrm{CH}_{3}\right)$. Anal. $\left(\mathrm{C}_{29} \mathrm{H}_{35} \mathrm{O}_{7} \mathrm{~N}_{3} \mathrm{~S}\right) \mathrm{C}, \mathrm{H}, \mathrm{N}$.

3.1.15. 2-(N,N-Diethylaminocarbonyloxymethyl)-4-(1Hpyrrole-2-ylcarbonyl)-1-(3,4,5-trimethoxybenzoyl)piperazine (4d). Compound 4d was prepared using pyrrole-2carboxylic acid $(202 \mathrm{mg})$, DIC $(335 \mu \mathrm{L}), \mathbf{2}$ (670 mg, 
$1.65 \mathrm{mmol}$ ) and obtained as a white solid (550 mg, $67 \%$ ) after a silica gel column chromatography using $\mathrm{MeOH} / \mathrm{CH}_{2} \mathrm{Cl}_{2}(1: 99, \mathrm{v} / \mathrm{v})$ as eluent: $R_{\mathrm{f}} 0.2\left(\mathrm{CH}_{2} \mathrm{Cl}_{2} /\right.$ $\mathrm{MeOH}, 95: 5$, v/v); $\mathrm{mp} 133{ }^{\circ} \mathrm{C}$; IR $\left(v \mathrm{~cm}^{-1}\right) 3299$ (NH), 1695 ( $\mathrm{C}=\mathrm{O}$ carbamate), 1630 ( $\mathrm{C}=\mathrm{O}$ amide), $1583(\mathrm{ArC}=\mathrm{C}) ;{ }^{1} \mathrm{H} \mathrm{NMR}\left(\mathrm{CDCl}_{3}, \delta\right): 9.50$ (br s, $1 \mathrm{H}, \mathrm{NH}), 6.92$ (m, 1H, ArH), 6.64 (s, 2H, ArH), $6.51(\mathrm{~m}, 1 \mathrm{H}, \mathrm{ArH}), 6.23(\mathrm{~m}, 1 \mathrm{H}, \mathrm{ArH}), 5.00-4.27$ $\left(\mathrm{m}, 3 \mathrm{H}, \mathrm{CH}_{2} \mathrm{OC}=\mathrm{O}\right.$ and piperazine $), 4.23-3.90(\mathrm{~m}$, $2 \mathrm{H}$, piperazine $), 3.86\left(\mathrm{~m}, 9 \mathrm{H}, \mathrm{OCH}_{3}\right), 3.80,2.98$ $\left(2 \mathrm{~m}, 8 \mathrm{H}, \mathrm{NCH}_{2} \mathrm{CH}_{3}\right.$ and piperazine $), 1.06(\mathrm{t}, 6 \mathrm{H}$, $\left.J=7 \mathrm{~Hz}, \mathrm{NCH}_{2} \mathrm{CH}_{3}\right) ;{ }^{13} \mathrm{C} \mathrm{NMR}\left(\mathrm{CDCl}_{3}, \delta\right): 170.64$, $162.62(\mathrm{C}=\mathrm{O}$ amide $), \quad 155.00 \quad(\mathrm{C}=\mathrm{O}$ carbamate $)$, $153.30,139.44,130.43123 .65,123.67,112.30,109.54$, 104.39 ( $\mathrm{ArC}=\mathrm{C}), 61.39 \quad\left(\mathrm{CH}_{2} \mathrm{OC}=\mathrm{O}\right), \quad 60.81, \quad 56.18$ $\left(\mathrm{CH}_{3} \mathrm{O}\right), 41.75, \quad 41.10 \quad\left(\mathrm{NCH}_{2} \mathrm{CH}_{3}\right), \quad 13.85, \quad 13.30$ $\left(\mathrm{NCH}_{2} \mathrm{CH}_{3}\right)$. Anal. $\left(\mathrm{C}_{25} \mathrm{H}_{34} \mathrm{O}_{7} \mathrm{~N}_{4}\right) \mathrm{C}, \mathrm{H}, \mathrm{N}$.

3.1.16. 4-(5-Chloro-1 $H$-indole-2-ylcarbonyl)-2-( $N, N-$ diethylaminocarbonyloxymethyl)-1-(3,4,5-trimethoxybenzoyl)piperazine (4e). Compound $4 \mathbf{e}$ was prepared using 5-chloro- $1 H$-indole-2-carboxylic acid (300 mg), DIC $(239 \mu \mathrm{L}), 2$ (570 $\mathrm{mg}, 2.44 \mathrm{mmol})$ and obtained as a white solid (450 g, 55\%) after a silica gel column chromatography using $\mathrm{MeOH} / \mathrm{CH}_{2} \mathrm{Cl}_{2}(1: 99$, v/v) as eluent: $R_{\mathrm{f}} 0.32\left(\mathrm{CH}_{2} \mathrm{Cl}_{2} / \mathrm{MeOH}, 95: 5, \mathrm{v} / \mathrm{v}\right) ; \mathrm{mp} 160{ }^{\circ} \mathrm{C}$; IR $(v$ $\left.\mathrm{cm}^{-1}\right) \quad 3198$ (NH), $1694 \quad(\mathrm{C}=\mathrm{O}$ carbamate), 1611 $(\mathrm{C}=\mathrm{O}$ amide), 1585 (ArC $=\mathrm{C}) ;{ }^{1} \mathrm{H} \mathrm{NMR}\left(\mathrm{CDCl}_{3}, \delta\right)$ : 9.95 (br s, 1H, NH), 7.54 (s, 1H, ArH), $7.36(\mathrm{~d}, 1 \mathrm{H}$, $J=8.75 \mathrm{~Hz}, \mathrm{ArH}), 7.22(\mathrm{~d}, 1 \mathrm{H}, J=8.75 \mathrm{~Hz}, \mathrm{ArH})$, $6.64(\mathrm{~s}, 3 \mathrm{H}, \mathrm{ArH}), 5.20-4.00\left(\mathrm{~m}, 5 \mathrm{H}, \quad \mathrm{CH}_{2} \mathrm{OC}=\mathrm{O}\right.$ and piperazine), $3.85\left(\mathrm{~s}, 9 \mathrm{H}, \mathrm{OCH}_{3}\right), 3.70-2.90(\mathrm{~m}$, $8 \mathrm{H}, \mathrm{NCH}_{2} \mathrm{CH}_{3}$ and piperazine), 1.07, $0.99(\mathrm{~m}, 6 \mathrm{H}$, $\left.\mathrm{N}\left(\mathrm{CH}_{2} \mathrm{CH}_{3}\right)_{2}\right) ;{ }^{13} \mathrm{C} \mathrm{NMR}\left(\mathrm{CDCl}_{3}, \delta\right): 170.71,163.14$ $(\mathrm{C}=\mathrm{O}$ amide $), \quad 155.07 \quad(\mathrm{C}=\mathrm{O}$ carbamate $), 153.36$, $139.55,134.25, \quad 130.21,129.69,128.07, \quad 126.12$, $124.92, \quad 120.87, \quad 113.01, \quad 104.54,104.40 \quad(\mathrm{ArC}=\mathrm{C})$, $61.02\left(\mathrm{CH}_{2} \mathrm{OC}=\mathrm{O}\right), \quad 60.83, \quad 56.21 \quad\left(\mathrm{CH}_{3} \mathrm{O}\right), 41.80$, $40.09\left(\mathrm{NCH}_{2} \mathrm{CH}_{3}\right), 13.86,13.29\left(\mathrm{NCH}_{2} \mathrm{CH}_{3}\right)$. Anal. $\left(\mathrm{C}_{29} \mathrm{H}_{35} \mathrm{O}_{7} \mathrm{~N}_{4} \mathrm{Cl}\right) \mathrm{C}, \mathrm{H}, \mathrm{N}$.

3.1.17. 2-( $N, N$-Diethylaminocarbonyloxymethyl)-4-(5methoxy-1 $H$-indole-2-ylcarbonyl)-1-(3,4,5-trimethoxybenzoyl)piperazine (4f). Compound $\mathbf{4 f}$ was prepared using 5-methoxy-1 $H$-indole-2-carboxylic acid $(308 \mathrm{mg})$, DIC $(251 \mu \mathrm{L}), 2(610 \mathrm{mg}, 1.61 \mathrm{mmol})$ and obtained as a white solid $(700 \mathrm{mg}, 75 \%)$ after a silica gel column chromatography using $\mathrm{CH}_{2} \mathrm{Cl}_{2}$ as eluent: $R_{\mathrm{f}} 0.4$ $\left(\mathrm{CH}_{2} \mathrm{Cl}_{2} / \mathrm{MeOH}, 95: 5, \mathrm{v} / \mathrm{v}\right) ; \mathrm{mp} 192{ }^{\circ} \mathrm{C}$; IR $\left(v \mathrm{~cm}^{-1}\right)$ $3283(\mathrm{NH}), 1694$ ( $\mathrm{C}=\mathrm{O}$ carbamate), 1626 ( $\mathrm{C}=\mathrm{O}$ amide $)$, $1585(\mathrm{ArC}=\mathrm{C}) ;{ }^{1} \mathrm{H} \mathrm{NMR}\left(\mathrm{CDCl}_{3}, \delta\right): 9.22$ (br s, $1 \mathrm{H}$, $\mathrm{NH}), 7.28$ (d, $1 \mathrm{H}, J=8.8 \mathrm{~Hz}, \mathrm{ArH}), 7.01-6.92(\mathrm{~m}, 2 \mathrm{H}$, ArH), 6.68 (s, 1H, ArH), 6.65 (s, 2H, ArH), 4.70-4.19 $\left(\mathrm{m}, 3 \mathrm{H}, \mathrm{CH}_{2} \mathrm{OC}=\mathrm{O}\right.$ and piperazine $), 4.05(\mathrm{~m}, 1 \mathrm{H}$, piperazine ), 3.86, $2.80\left(2 \mathrm{~s}, 12 \mathrm{H}, \mathrm{OCH}_{3}\right), 3.77-3.30(\mathrm{~m}, 3 \mathrm{H}$, piperazine), $3.17-2.77\left(\mathrm{~m}, 6 \mathrm{H}, \mathrm{NCH}_{2} \mathrm{CH}_{3}\right.$ and piperazine), $1.02\left(\mathrm{~m}, 6 \mathrm{H}, \mathrm{NCH}_{2} \mathrm{CH}_{3}\right) ;{ }^{13} \mathrm{C} \mathrm{NMR}\left(\mathrm{CDCl}_{3}, \delta\right)$ : $171.11,163.60(\mathrm{C}=\mathrm{O}$ amide $), 154.68(\mathrm{C}=\mathrm{O}$ carbamate $)$, $153.39,139.67,131.35,129.85,128.39,127.54(\mathrm{ArC}=\mathrm{C})$, $116.16,112.80,105.36,104.44,102.17(\mathrm{ArC}=\mathrm{C}), 61.22$ $\left(\mathrm{CH}_{2} \mathrm{OC}=\mathrm{O}\right), 60.86,56.24,55.65\left(\mathrm{CH}_{3} \mathrm{O}\right) 41.89,41.19$ $\left(\mathrm{NCH}_{2} \mathrm{CH}_{3}\right), \quad 13.85, \quad 13.28 \quad\left(\mathrm{NCH}_{2} \mathrm{CH}_{3}\right)$. Anal. $\left(\mathrm{C}_{30} \mathrm{H}_{38} \mathrm{O}_{8} \mathrm{~N}_{4}\right) \mathrm{C}, \mathrm{H}, \mathrm{N}$.
3.1.18. 2-(N,N-Diethylaminocarbonyloxymethyl)-1-(1methyl-1 $H$-indole-2-ylcarbonyl)-4-(3,4,5-trimethoxybenzoyl)piperazine (3k). A mixture of 3a (590 mg, $1.06 \mathrm{mmol})$, iodomethane $(520 \mu \mathrm{L}, 10.6 \mathrm{mmol})$ and $\mathrm{K}_{2} \mathrm{CO}_{3}(1.46 \mathrm{~g}, 10.6 \mathrm{mmol})$ in acetonitrile $(25 \mathrm{~mL})$ was refluxed for $24 \mathrm{~h}$. After evaporation, the residue was taken in $\mathrm{CH}_{2} \mathrm{Cl}_{2}(25 \mathrm{~mL})$ and then washed with water, dried $\left(\mathrm{MgSO}_{4}\right)$ and filtered, and the solvent was removed in vacuo. The product was isolated as a solid (380 mg, 63\%) after a silica gel column chromatography using $\mathrm{CH}_{2} \mathrm{Cl}_{2}$ as eluent: $R_{\mathrm{f}} 0.47\left(\mathrm{MeOH} / \mathrm{CH}_{2} \mathrm{Cl}_{2}, 5: 95\right.$, $\mathrm{v} / \mathrm{v}) ; \mathrm{mp} 120^{\circ} \mathrm{C}$; IR $\left(v \mathrm{~cm}^{-1}\right) 1691(\mathrm{C}=\mathrm{O}$ carbamate), $1628\left(\mathrm{C}=\mathrm{O}\right.$ amide), $1585(\mathrm{ArC}=\mathrm{C}) ;{ }^{1} \mathrm{H} \mathrm{NMR}\left(\mathrm{CDCl}_{3}\right.$, $\delta): 7.55(\mathrm{~d}, 1 \mathrm{H}, J=7.9 \mathrm{~Hz}, \mathrm{ArH}), 7.24(\mathrm{~m}, 2 \mathrm{H}, \mathrm{ArH})$, $7.11(\mathrm{~m}, 1 \mathrm{H}, \mathrm{ArH}), 6.58(\mathrm{~s}, 3 \mathrm{H}, \mathrm{ArH}), 5.1(\mathrm{~m}, 1 \mathrm{H}$, $\left.\mathrm{CH}_{2} \mathrm{OC}=\mathrm{O}\right), 4.80-3.95\left(\mathrm{~m}, 4 \mathrm{H}, \mathrm{CH}_{2} \mathrm{OC}=\mathrm{O}\right.$ and piperazine), 3.79 (m, 12H, $\left.\mathrm{OCH}_{3}, \mathrm{NCH}_{3}\right), 3.70-2.70(\mathrm{~m}, 8 \mathrm{H}$, $\mathrm{NCH}_{2} \mathrm{CH}_{3}$ and piperazine), 1.02, $0.99(2 \mathrm{~m}, 6 \mathrm{H}$, $\left.\mathrm{NCH}_{2} \mathrm{CH}_{3}\right) ;{ }^{13} \mathrm{C}$ NMR $\left(\mathrm{CDCl}_{3}, \delta\right): 170.86,163.81$ ( $\mathrm{C}=\mathrm{O}$ amide $), \quad 155.05 \quad(\mathrm{C}=\mathrm{O}$ carbamate $), 153.39$, $139.37,138.08,130.62,130.12,126.09,123.72,121.64$, $120.41, \quad 109.85, \quad 104.28, \quad 104.20 \quad(\mathrm{ArC}=\mathrm{C}), \quad 60.83$ $\left(\mathrm{CH}_{2} \mathrm{OC}=\mathrm{O}\right), \quad 60.78, \quad 56.25 \quad\left(\mathrm{CH}_{3} \mathrm{O}\right), \quad 41.84, \quad 41.12$ $\left(\mathrm{NCH}_{2} \mathrm{CH}_{3}\right), \quad 13.84, \quad 13.33 \quad\left(\mathrm{NCH}_{2} \mathrm{CH}_{3}\right)$. Anal. $\left(\mathrm{C}_{30} \mathrm{H}_{38} \mathrm{O}_{7} \mathrm{~N}_{4}\right) \mathrm{C}, \mathrm{H}, \mathrm{N}$.

3.1.19. 2-( $N, N$-Diethylaminocarbonyloxymethyl)-4-(1methyl-1 $H$-indole-2-ylcarbonyl)-1-(3,4,5-trimethoxybenzoyl)piperazine (4k). Compound $\mathbf{4 k}$ was prepared using the same procedure as for $\mathbf{3 k}$ but from $\mathbf{4 a}(130 \mathrm{mg}$, $0.235 \mathrm{mmol})$, iodomethane $(117 \mu \mathrm{L}), \mathrm{K}_{2} \mathrm{CO}_{3}(63 \mathrm{mg})$ and obtained as a white solid (130 mg, 98\%) after a silica gel column chromatography using $\mathrm{CH}_{2} \mathrm{Cl}_{2}$ as eluent: $R_{\mathrm{f}}$ $0.5\left(\mathrm{MeOH} / \mathrm{CH}_{2} \mathrm{Cl}_{2}, 5: 95, \mathrm{v} / \mathrm{v}\right) ; \mathrm{mp} 86^{\circ} \mathrm{C} ; \mathrm{IR}\left(v \mathrm{~cm}^{-1}\right)$ $1696(\mathrm{C}=\mathrm{O}$ carbamate), $1626(\mathrm{C}=\mathrm{O}$ amide $), 1582$ $(\mathrm{ArC}=\mathrm{C}) ;{ }^{1} \mathrm{H} \quad \mathrm{NMR} \quad\left(\mathrm{CDCl}_{3}, \delta\right): 7.53 \quad(\mathrm{~d}, \quad 1 \mathrm{H}$, $J=7.9 \mathrm{~Hz}, \mathrm{ArH}), 7.27$ (m, 2H, ArH), 7.07 (m, 1H, $\mathrm{ArH}), 6.58$ (m, 3H, ArH), 5.10-3.95 (m, 5H, $\mathrm{CH}_{2} \mathrm{OC}=\mathrm{O}$ and piperazine $), 3.79\left(\mathrm{~m}, 12 \mathrm{H}, \mathrm{OCH}_{3}\right.$ and $\left.\mathrm{NCH}_{3}\right), 3.60$ $2.50\left(\mathrm{~m}, 8 \mathrm{H}, \mathrm{NCH}_{2} \mathrm{CH}_{3}\right.$ and piperazine), 0.98, $0.81(2 \mathrm{~m}$, $\left.6 \mathrm{H}, \mathrm{NCH}_{2} \mathrm{CH}_{3}\right) ;{ }^{13} \mathrm{C} \mathrm{NMR}\left(\mathrm{CDCl}_{3}, \delta\right): 170.70,163.93$ $(\mathrm{C}=\mathrm{O}$ amide $), \quad 155.04 \quad(\mathrm{C}=\mathrm{O}$ carbamate $), 153.35$, $139.08,130.37,130.23,126.38,123.64,121.59,120.38$, $109.86,104.37,104.16(\mathrm{ArC}=\mathrm{C}), 60.58\left(\mathrm{CH}_{2} \mathrm{OC}=\mathrm{O}\right)$, 60.83, 56.21 $\left(\mathrm{CH}_{3} \mathrm{O}\right), 41.83,41.02\left(\mathrm{NCH}_{2} \mathrm{CH}_{3}\right), 13.74$, $13.39\left(\mathrm{NCH}_{2} \mathrm{CH}_{3}\right)$. Anal. $\left(\mathrm{C}_{30} \mathrm{H}_{38} \mathrm{O}_{7} \mathrm{~N}_{4}\right) \mathrm{C}, \mathrm{H}, \mathrm{N}$.

3.1.20. 2-(N,N-Diethylaminocarbonyloxymethyl)-1-(5-nitro-1 $H$-indole-2-ylcarbonyl)-1-(3,4,5-trimethoxybenzoyl)piperazine (6a). A solution of 5-nitro- $1 H$-indole-2carboxylic acid $\mathbf{5}^{21}(181 \mathrm{mg}, 0.88 \mathrm{mmol})$ in thionyl chloride $(147 \mu \mathrm{L}, 2 \mathrm{mmol})$ was stirred in dichloromethane $(20 \mathrm{~mL})$ under reflux for one night. The solvent was then evaporated under reduced pressure and a solution of the amine 1 (300 mg, $0.73 \mathrm{mmol})$ and triethylamine $(205 \mu \mathrm{L}$, $1.42 \mathrm{mmol})$ in dichloromethane $(40 \mathrm{~mL})$ was added. The mixture was stirred at room temperature for $12 \mathrm{~h}$. The reaction mixture was then washed with saturated $\mathrm{NaHCO}_{3}$ and water, dried $\left(\mathrm{MgSO}_{4}\right)$, filtered and the solvent removed in vacuo. The compound $6 \mathbf{a}$ was obtained after a silica gel column chromatography using $\mathrm{MeOH} /$ $\mathrm{CH}_{2} \mathrm{Cl}_{2}(1: 99$, v/v) as eluent, as a yellow powder (222 mg, 51\%): $R_{\mathrm{f}} 0.30\left(\mathrm{CH}_{2} \mathrm{Cl}_{2} / \mathrm{MeOH}, 95: 5, \mathrm{v} / \mathrm{v}\right) ; \mathrm{mp}$ 
$120^{\circ} \mathrm{C}$; IR $\left(v \mathrm{~cm}^{-1}\right) 3262(\mathrm{NH}), 2943(\mathrm{ArCH}), 1697$ $(\mathrm{C}=\mathrm{O}$ carbamate $), 1632,1619(\mathrm{C}=\mathrm{O}$ amide $), 1583$ $(\mathrm{ArC}=\mathrm{C}) ;{ }^{1} \mathrm{H}$ NMR $\left(\mathrm{CDCl}_{3}, \delta\right): 10.41$ (br s, $\left.1 \mathrm{H}, \mathrm{NH}\right)$, $8.6(\mathrm{~s}, 1 \mathrm{H}, \operatorname{ArH}), 8.14(\mathrm{~d}, 1 \mathrm{H}, J=9 \mathrm{~Hz}, \operatorname{ArH}), 7.47(\mathrm{~d}$, $1 \mathrm{H}, J=9 \mathrm{~Hz}, \operatorname{ArH}), 6.87(\mathrm{~s}, 1 \mathrm{H}, \mathrm{ArH}), 6.64(\mathrm{~s}, 2 \mathrm{H}$, $\mathrm{ArH}), 5.40,4.10\left(\mathrm{~m}, 6 \mathrm{H}, \mathrm{CH}_{2} \mathrm{OC}=\mathrm{O}\right.$ and piperazine $)$, $3.87\left(\mathrm{~s}, 9 \mathrm{H}, \mathrm{OCH}_{3}\right), 3.60,2.70\left(2 \mathrm{~m}, 7 \mathrm{H}, \mathrm{NCH}_{2} \mathrm{CH}_{3}\right.$ and piperazine), $0.88,1.01\left(2 \mathrm{~m}, 6 \mathrm{H}, \mathrm{NCH}_{2} \mathrm{CH}_{3}\right) ;{ }^{13} \mathrm{C}$ NMR $\left(\mathrm{CDCl}_{3}, \delta\right): 170.75,162.43(\mathrm{C}=\mathrm{O}$ amide $), 154.87$ $(\mathrm{C}=\mathrm{O}$ carbamate $), 153.21,141.98,139.31,138.75$, $131.70,129.74,126.09,119.30,119.06,112.06,107.52$, $104.20 \quad(\mathrm{ArC}=\mathrm{C}), 60.73 \quad\left(\mathrm{CH}_{2} \mathrm{OC}=\mathrm{O}\right), 60.62, \quad 56.07$ $\left(\mathrm{CH}_{3} \mathrm{O}\right), 41.72,40.92 \quad\left(\mathrm{NCH}_{2} \mathrm{CH}_{3}\right), 13.56,13.05$ $\left(\mathrm{NCH}_{2} \mathrm{CH}_{3}\right)$. Anal. $\left(\mathrm{C}_{29} \mathrm{H}_{38} \mathrm{O}_{8} \mathrm{~N}_{4}\right) \mathrm{C}, \mathrm{H}, \mathrm{N}$.

3.1.21. 2-( $N, N$-Diethylaminocarbonyloxymethyl)-4-(5-nitro-1H-indole-2-ylcarbonyl)-1-(3,4,5-trimethoxybenzoyl)piperazine (6b). Compound $6 \mathbf{b}$ was prepared using the same procedure as for $\mathbf{6 a}$ from 5-nitro-indole-2-carboxylic acid $\mathbf{5}^{21}(258 \mathrm{mg}, 1.46 \mathrm{mmol})$, thionyl chloride $(220 \mu \mathrm{L}, 3 \mathrm{mmol})$ and the amine $2(500 \mathrm{mg}, 1.22 \mathrm{mmol})$ in the presence of triethylamine $(300 \mu \mathrm{L}, 2.16 \mathrm{mmol})$. The compound $\mathbf{6 b}$ was obtained after a silica gel column chromatography using $\mathrm{MeOH} / \mathrm{CH}_{2} \mathrm{Cl}_{2}(1: 99$, v/v) as eluent, as a yellow solid $(473 \mathrm{mg}, 65 \%): R_{\mathrm{f}} 0.32\left(\mathrm{CH}_{2} \mathrm{Cl}_{2} /\right.$ $\mathrm{MeOH}, 95: 5, \mathrm{v} / \mathrm{v}) ; \mathrm{mp} 111.2{ }^{\circ} \mathrm{C}$; IR $\left(v \mathrm{~cm}^{-1}\right) 3438$ $(\mathrm{NH}), 3054(\mathrm{ArCH}), 1694(\mathrm{C}=\mathrm{O}$ carbamate $), 1633$ $(\mathrm{C}=\mathrm{O}$ amide $), 1584(\mathrm{ArC}=\mathrm{C}) ;{ }^{1} \mathrm{H}$ NMR $\left(\mathrm{CDCl}_{3}, \delta\right)$ : 10.93 (br s, 1H, NH), 8.50 (s, 1H, ArH), 8.04 (d, 1H, $J=9 \mathrm{~Hz}, \operatorname{ArH}), 7.14(\mathrm{~d}, 1 \mathrm{H}, J=9 \mathrm{~Hz}, \operatorname{ArH}), 6.87$ (s, $1 \mathrm{H}, \operatorname{ArH}), 6.64(\mathrm{~s}, 2 \mathrm{H}, \mathrm{ArH}), 4.00-5.40(\mathrm{~m}, 5 \mathrm{H}$, $\mathrm{CH}_{2} \mathrm{OC}=\mathrm{O}$ and piperazine), $3.81\left(\mathrm{~s}, 9 \mathrm{H}, \mathrm{OCH}_{3}\right), 3.70$, $2.00\left(2 \mathrm{~m}, 8 \mathrm{H}, \mathrm{NCH}_{2} \mathrm{CH}_{3}\right.$ and piperazine), $0.60-1.10$ $\left(\mathrm{m}, 6 \mathrm{H}, \mathrm{NCH}_{2} \mathrm{CH}_{3}\right) ;{ }^{13} \mathrm{C}$ NMR $\left(\mathrm{CDCl}_{3}, \delta\right): 170.71$, $162.68 \quad(\mathrm{C}=\mathrm{O}$ amide $), \quad 155.00 \quad(\mathrm{C}=\mathrm{O}$ carbamate $)$, $153.29,142.12,139.55,138.78,131.70,130.00,126.00$, $119.32,118.96,112.15,106.81,104.39(\mathrm{ArC}=\mathrm{C}), 60.91$ $\left(\mathrm{CH}_{2} \mathrm{OC}=\mathrm{O}\right), \quad 60.74, \quad 56.14 \quad\left(\mathrm{CH}_{3} \mathrm{O}\right), 41.74, \quad 41.03$ $\left(\mathrm{NCH}_{2} \mathrm{CH}_{3}\right), \quad 13.79, \quad 13.19 \quad\left(\mathrm{NCH}_{2} \mathrm{CH}_{3}\right)$. Anal. $\left(\mathrm{C}_{29} \mathrm{H}_{38} \mathrm{O}_{8} \mathrm{~N}_{4}\right) \mathrm{C}, \mathrm{H}, \mathrm{N}$.

3.1.22. 2-( $N, N$-Diethylaminocarbonyloxymethyl)-1-[(1Hindole-3-yl)oxoacetyl]-1-(3,4,5-trimethoxybenzoyl)piperazine (8). To a mixture of $1(500 \mathrm{mg}, 1.22 \mathrm{mmol})$ and triethylamine $(200 \mu \mathrm{L}, 1.46 \mathrm{mmol})$ in dichloromethane $(70 \mathrm{~mL})$ was gradually added $\alpha$-oxo- $1 H$-indole-3-ylacetyl chloride $7^{22}$ (303 mg, $1.46 \mathrm{mmol}$ ). The reaction mixture was stirred for $48 \mathrm{~h}$ and then washed with water, dried $\left(\mathrm{MgSO}_{4}\right)$, filtered, and the solvent removed in vacuo. The product was isolated as a white solid $(380 \mathrm{mg}$, $51 \%$ ) after a silica gel column chromatography using $\mathrm{MeOH} / \mathrm{CH}_{2} \mathrm{Cl}_{2}\left(1: 99\right.$, v/v) as eluent: $R_{\mathrm{f}} 0.24(\mathrm{MeOH} /$ $\mathrm{CH}_{2} \mathrm{Cl}_{2}, 5: 95$, v/v); $\mathrm{mp} 154{ }^{\circ} \mathrm{C}$; IR $\left(v \mathrm{~cm}^{-1}\right) 3259$ $(\mathrm{NH}), 1692(\mathrm{C}=\mathrm{O}$ carbamate $), 1629(\mathrm{C}=\mathrm{O}$ amide $)$, $1581(\mathrm{ArC}=\mathrm{C}) ;{ }^{1} \mathrm{H}$ NMR $\left(\mathrm{CDCl}_{3}, \delta\right): 10.52(\mathrm{br} \mathrm{s}, 1 \mathrm{H}$, $\mathrm{NH}), 8.26(\mathrm{~m}, 1 \mathrm{H}, \mathrm{ArH}), 7.86(\mathrm{~m}, 1 \mathrm{H}, \mathrm{ArH}), 7.39(\mathrm{~s}$, $1 \mathrm{H}, \mathrm{ArH}), 7.28(\mathrm{~m}, 2 \mathrm{H}, \mathrm{ArH}), 6.60(\mathrm{~s}, 2 \mathrm{H}, \mathrm{ArH})$, 5.20-3.98 (m, 5H, $\mathrm{CH}_{2} \mathrm{OC}=\mathrm{O}$ and piperazine), $3.83(\mathrm{~s}$, $\left.9 \mathrm{H}, \mathrm{OCH}_{3}\right), 3.70,2.80\left(2 \mathrm{~m}, 8 \mathrm{H}, \mathrm{NCH}_{2} \mathrm{CH}_{3}\right.$ and piperazine), $0.99,0.80\left(2 \mathrm{~m}, 6 \mathrm{H}, \quad \mathrm{NCH}_{2} \mathrm{CH}_{3}\right) ;{ }^{13} \mathrm{C} \mathrm{NMR}$ $\left(\mathrm{CDCl}_{3}, \delta\right): 184.75,170.00,167.16(\mathrm{C}=\mathrm{O}), 155.17$ $(\mathrm{C}=\mathrm{O}$ carbamate $), 153.37, \quad 139.46, \quad 136.78,129.87$, $125.27,114.37,136.69,124.28,123.28,121.77,112.07$,
104.27 $(\mathrm{ArC}=\mathrm{C}), \quad 60.81 \quad\left(\mathrm{CH}_{2} \mathrm{OC}=\mathrm{O}\right), \quad 60.56, \quad 56.22$ $\left(\mathrm{CH}_{3} \mathrm{O}\right), \quad 41.94, \quad 41.27 \quad\left(\mathrm{NCH}_{2} \mathrm{CH}_{3}\right), \quad 13.88, \quad 13.31$ $\left(\mathrm{NCH}_{2} \mathrm{CH}_{3}\right)$. Anal. $\left(\mathrm{C}_{30} \mathrm{H}_{36} \mathrm{O}_{8} \mathrm{~N}_{4}\right) \mathrm{C}, \mathrm{H}, \mathrm{N}$.

3.1.23. 2-(N,N-Diethylaminocarbonyloxymethyl)-1-(1Hindole-1-ylcarbonyl)-4-(3,4,5-trimethoxybenzoyl)piperazine (10). To a mixture of indole-1-carboxylic acid $\mathbf{9}^{23}$ $(1.25 \mathrm{~g}, \quad 7.75 \mathrm{mmol})$ dissolved in dichloromethane ( $20 \mathrm{~mL})$ was added DIC $(720 \mu \mathrm{L}, 4.65 \mathrm{mmol})$. The reaction mixture was stirred for $20 \mathrm{~min}$ at room temperature. A solution of $1(1.58 \mathrm{~g}, 3.87 \mathrm{mmol})$ dissolved in dichloromethane $(15 \mathrm{~mL})$ was then added to the mixture and stirred at room temperature for $12 \mathrm{~h}$. The solution was then washed with water, dried $\left(\mathrm{MgSO}_{4}\right)$, filtered and the solvent removed in vacuo. The product was isolated as a white solid $(2.11 \mathrm{~g}, 99 \%)$ after a silica gel column chromatography using $\mathrm{MeOH} / \mathrm{CH}_{2} \mathrm{Cl}_{2}(1: 99$, v/v) as eluent: $R_{\mathrm{f}} 0.3\left(\mathrm{MeOH} / \mathrm{CH}_{2} \mathrm{Cl}_{2}, 5: 95, \mathrm{v} / \mathrm{v}\right) ; \mathrm{mp} 62^{\circ} \mathrm{C}$; IR $\left(v \mathrm{~cm}^{-1}\right) 1700(\mathrm{C}=\mathrm{O}$ carbamate $), 1635(\mathrm{C}=\mathrm{O}$ amide $)$, $1596(\mathrm{ArC}=\mathrm{C}) ;{ }^{1} \mathrm{H}$ NMR $\left(\mathrm{CDCl}_{3}, \delta\right): 7.64(\mathrm{~d}, 1 \mathrm{H}$, $J=7.9 \mathrm{~Hz}, \operatorname{ArH}), 7.49(\mathrm{~d}, 1 \mathrm{H}, J=8 \mathrm{~Hz}, \operatorname{ArH}), 7.25$ $7.05(\mathrm{~m}, 3 \mathrm{H}, \operatorname{ArH}), 6.56(\mathrm{~s}, 2 \mathrm{H}, \operatorname{ArH}), 6.49(\mathrm{~m}, 1 \mathrm{H}$, $\mathrm{ArH}), 4.70-3.90\left(\mathrm{~m}, 5 \mathrm{H}, \mathrm{CH}_{2} \mathrm{OC}=\mathrm{O}\right.$ and piperazine $)$, 3.76, $3.75\left(2 \mathrm{~s}, 9 \mathrm{H}, \mathrm{OCH}_{3}\right), 3.70-3.00\left(\mathrm{~m}, 8 \mathrm{H}, \mathrm{NCH}_{2} \mathrm{CH}_{3}\right.$ and piperazine), $1.00,0.95\left(2 \mathrm{~m}, 6 \mathrm{H}, \mathrm{NCH}_{2} \mathrm{CH}_{3}\right) ;{ }^{13} \mathrm{C}$ NMR $\left(\mathrm{CDCl}_{3}, \delta\right): 170.38(\mathrm{C}=\mathrm{O}$ amide $), 156.96(\mathrm{C}=\mathrm{O}$ carbamate $), 154.70 \quad(\mathrm{C}=\mathrm{O}$ ureate $), 153.99,153.04$, $139.08,135.08,129.68,129.24,125.56,123.43,212.86$, $120.71, \quad 113.04, \quad 106.15, \quad 103.95 \quad(\mathrm{ArC}=\mathrm{C}), \quad 60.62$ $\left(\mathrm{CH}_{2} \mathrm{OC}=\mathrm{O}\right), \quad 60.41, \quad 55.88 \quad\left(\mathrm{CH}_{3} \mathrm{O}\right), \quad 41.19, \quad 40.81$ $\left(\mathrm{NCH}_{2} \mathrm{CH}_{3}\right), \quad 13.46, \quad 12.94 \quad\left(\mathrm{NCH}_{2} \mathrm{CH}_{3}\right)$. Anal. $\left(\mathrm{C}_{29} \mathrm{H}_{36} \mathrm{O}_{7} \mathrm{~N}_{4} \cdot 0.05 \mathrm{H}_{2} \mathrm{O}\right) \mathrm{C}, \mathrm{H}, \mathrm{N}$.

3.1.24. $2-(\mathrm{N}, \mathrm{N}$-Diethylaminocarbonyloxymethyl)-1-(1 Hindole-2-ylcarbonyl)-4-(triphenylmethyl)piperazine (12). Compound 12 was prepared using the same procedure as for 3 from $\mathbf{1 1}^{20}(2.05 \mathrm{~g}, 3.14 \mathrm{mmol}), 1 \mathrm{H}$-indole-2-carboxylic acid $(854.5 \mathrm{mg})$, DIC $(826 \mu \mathrm{L})$ and isolated as a white solid $(2.12 \mathrm{~g}, 85 \%)$ after a silica gel column chromatography using $\mathrm{MeOH} / \mathrm{CH}_{2} \mathrm{Cl}_{2}(1: 99$, v/v) as eluent: $R_{\mathrm{f}} 0.3\left(\mathrm{MeOH} / \mathrm{CH}_{2} \mathrm{Cl}_{2}, 2: 98, \mathrm{v} / \mathrm{v}\right) ; \mathrm{mp} 134{ }^{\circ} \mathrm{C}$; IR $(v$ $\left.\mathrm{cm}^{-1}\right) 3275(\mathrm{NH}), 2974(\mathrm{ArCH}), 1690(\mathrm{C}=\mathrm{O}$ carbamate), $1599\left(\mathrm{C}=\mathrm{O}\right.$ amide), $1523(\mathrm{ArC}=\mathrm{C}) ;{ }^{1} \mathrm{H}$ NMR $\left(\mathrm{CDCl}_{3}, \delta\right): 10.10($ br s, $1 \mathrm{H}, \mathrm{NH}), 7.00-8.00(\mathrm{~m}, 19 \mathrm{H}$, $\mathrm{ArH}), 6.77(\mathrm{~s}, 1 \mathrm{H}, \mathrm{ArH}), 5.22\left(\mathrm{br} \mathrm{s}, 1 \mathrm{H}, \mathrm{CH}_{2} \mathrm{OC}=\mathrm{O}\right)$, $5.02\left(\right.$ br s, $\left.1 \mathrm{H}, \mathrm{CH}_{2} \mathrm{OC}=\mathrm{O}\right), 3.80-4.70(\mathrm{~m}, 3 \mathrm{H}$, piperazine), $2.80-3.50\left(\mathrm{~m}, 6 \mathrm{H}, \mathrm{NCH}_{2} \mathrm{CH}_{3}\right.$ and piperazine), 1.51-1.47 (m, 2H, piperazine $), 0.80-1.15(\mathrm{~m}, 6 \mathrm{H}$, $\left.\mathrm{NCH}_{2} \mathrm{CH}_{3}\right) ;{ }^{13} \mathrm{C} \quad \mathrm{NMR}\left(\mathrm{CDCl}_{3}, \delta\right): 162.84(\mathrm{C}=\mathrm{O}$ amide), $155.54(\mathrm{C}=\mathrm{O}$ carbamate $), 136.36,135.84$, $128.90,127.05,127.80,127.53,126.20,123.91,121.50$, $120.00,111.76,105.44(\mathrm{ArC}=\mathrm{C}), 76.81\left(\mathrm{C}(\mathrm{Ph})_{3}\right), 62.83$ $\left(\mathrm{CH}_{2} \mathrm{OC}=\mathrm{O}\right), 48.90,48.72,47.85\left(\mathrm{CH}_{2}\right.$ of piperazine $)$, 41.72, $41.05\left(\mathrm{NCH}_{2} \mathrm{CH}_{3}\right), 13.67,13.25\left(\mathrm{NCH}_{2} \mathrm{CH}_{3}\right)$.

3.1.25. 2-(N,N-Diethylaminocarbonyloxymethyl)-1-(1 Hindole-2-ylcarbonyl)piperazine (13). To a solution of 12 $(1.64 \mathrm{~g}, 2.7 \mathrm{mmol})$ in $\mathrm{MeOH}(30 \mathrm{~mL})$ was added dropwise trifluoroacetic acid (TFA, $3 \mathrm{~mL}$ ). This solution was stirred for $20 \mathrm{~min}$ at room temperature and the solvent removed in vacuo. The residue was then taken up with $\mathrm{CH}_{2} \mathrm{Cl}_{2}$, washed with saturated $\mathrm{NaHCO}_{3}$ solution and water, dried $\left(\mathrm{MgSO}_{4}\right)$, filtered and evaporated. 
The compound 13 was obtained as a wax (900 mg, 93\%) after a silica gel column chromatography using $\mathrm{CH}_{2} \mathrm{Cl}_{2}$ as eluent: $R_{\mathrm{f}} 0.6\left(\mathrm{CH}_{2} \mathrm{Cl}_{2} / \mathrm{MeOH}, 90: 10\right.$, v/v); IR $(v$ $\left.\mathrm{cm}^{-1}\right) 3276(\mathrm{NH}), 3054(\mathrm{ArCH}), 1692$ (C=O carbamate), 1604 ( $\mathrm{C}=\mathrm{O}$ amide), $1525(\mathrm{ArC}=\mathrm{C}) ;{ }^{1} \mathrm{H}$ NMR $\left(\mathrm{CDCl}_{3}, \delta\right): 9.76$ (br s, $\left.1 \mathrm{H}, \mathrm{NH}\right), 7.62(\mathrm{~d}, 1 \mathrm{H}, J=8 \mathrm{~Hz}$, ArH), $7.43(\mathrm{~d}, 1 \mathrm{H}, J=8 \mathrm{~Hz}, \operatorname{ArH}), 7.25(\mathrm{~m}, 1 \mathrm{H}$, ArH), 7.10 (m, 1H, ArH), 6.85 (s, 1H, ArH), 4.20, $5.20\left(2 \mathrm{~m}, 5 \mathrm{H}, \mathrm{CH}_{2} \mathrm{OC}=\mathrm{O}\right.$ and piperazine $), 2.60,3.85$ (2m, 9H, $\mathrm{NCH}_{2} \mathrm{CH}_{3}$ and piperazine), $1.16(\mathrm{~m}, 6 \mathrm{H}$, $\left.\mathrm{NCH}_{2} \mathrm{CH}_{3}\right) ;{ }^{13} \mathrm{C}$ NMR $\left(\mathrm{CDCl}_{3}, \delta\right): 163.30 \quad(\mathrm{C}=\mathrm{O}$ amide), $155.52(\mathrm{C}=\mathrm{O}$ carbamate), 135.84, 129.21, $127.29,124.11,121.72,120.27,111.75,105.36(\mathrm{ArC}=\mathrm{C})$, $61.69\left(\mathrm{CH}_{2} \mathrm{OC}=\mathrm{O}\right), 45.99\left(\mathrm{CH}_{2}\right.$ of piperazine $), 41.78$, $41.14\left(\mathrm{NCH}_{2} \mathrm{CH}_{3}\right), 13.79,13.28\left(\mathrm{NCH}_{2} \mathrm{CH}_{3}\right)$.

3.1.26. 3-( $\mathrm{N}, \mathrm{N}$-Diethylaminocarbonyloxymethyl)-1-(1 Hindole-2-ylcarbonyl)piperazine (15). Compound 15 was prepared using the same procedure as described for 3, from $\mathbf{1 4}^{24}$ (200 mg, $\left.0.69 \mathrm{mmol}\right)$, 2-indole carboxylic acid (134 mg, $0.69 \mathrm{mmol})$ and DCC $(175 \mu \mathrm{L}$, $0.69 \mathrm{mmol})$ in the presence of triethylamine $(194 \mu \mathrm{L}$, $1.38 \mathrm{mmol})$ and isolated as a wax $(230 \mathrm{~g}, 90 \%)$ after a silica gel column chromatography using $\mathrm{MeOH} /$ $\mathrm{CH}_{2} \mathrm{Cl}_{2}\left(2: 98\right.$, v/v) as eluent: $R_{\mathrm{f}} 0.63\left(\mathrm{MeOH} / \mathrm{CH}_{2} \mathrm{Cl}_{2}\right.$, $10: 90, \mathrm{v} / \mathrm{v}) ; \operatorname{IR}\left(v \mathrm{~cm}^{-1}\right) 3280(\mathrm{NH}), 3054(\mathrm{ArCH})$, 1694 ( $\mathrm{C}=\mathrm{O}$ carbamate), $1607 \quad(\mathrm{C}=\mathrm{O}$ amide $), 1530$ $(\mathrm{ArC}=\mathrm{C}) ;{ }^{1} \mathrm{H} \quad \mathrm{NMR} \quad\left(\mathrm{CDCl}_{3}, \delta\right): 9.73 \quad$ (br $\mathrm{s}$, $1 \mathrm{H}, \mathrm{NH}), 7.61(\mathrm{~d}, 1 \mathrm{H}, J=7.9 \mathrm{~Hz}, \mathrm{ArH}), 7.42(\mathrm{~d}$, $1 \mathrm{H}, J=7.9 \mathrm{~Hz}, \mathrm{ArH}), 7.22(\mathrm{~m}, 1 \mathrm{H}, \mathrm{ArH}), 7.14(\mathrm{~m}$, $1 \mathrm{H}, \mathrm{ArH}), 6.76(\mathrm{~s}, 1 \mathrm{H}, \mathrm{ArH}), 4.70,4.00(2 \mathrm{~m}, 4 \mathrm{H}$, $\mathrm{CH}_{2} \mathrm{OC}=\mathrm{O}$ and piperazine), 3.50, 2.60 (2m, 9H, $\mathrm{NCH}_{2} \mathrm{CH}_{3}$ and piperazine), 2.27 (br s, $1 \mathrm{H}, \mathrm{NH}$ ), $1.16\left(\mathrm{~m}, 6 \mathrm{H}, \mathrm{NCH}_{2} \mathrm{CH}_{3}\right) ;{ }^{13} \mathrm{C} \mathrm{NMR}\left(\mathrm{CDCl}_{3}, \delta\right)$ : $165.00 \quad(\mathrm{C}=\mathrm{O}$ amide $), 155.67 \quad(\mathrm{C}=\mathrm{O}$ carbamate $)$, $135.81, \quad 129.25, \quad 127.00, \quad 124.00,121.80,120.36$, 111.78, $106.00 \quad(\mathrm{ArC}=\mathrm{C}), 61.59 \quad\left(\mathrm{CH}_{2} \mathrm{OC}=\mathrm{O}\right), 45.63$ $\left(\mathrm{CH}_{2}\right.$ piperazine), 41.70, $41.21\left(\mathrm{NCH}_{2} \mathrm{CH}_{3}\right), 13.86$, $13.18\left(\mathrm{NCH}_{2} \mathrm{CH}_{3}\right)$.

3.1.27. General procedure for the synthesis of $16 a, b, 17 a, b$ and 18. A solution of 2,13 or 15 in dichloroethane (DCE) or dichloromethane, the aldehyde (1.1 or 1.2 equiv) and $\mathrm{NaBH}(\mathrm{OAc})_{3}$ (1.1 or 1.2 equiv) was stirred at room temperature until the reaction was complete (TLC monitored). The solvent was removed under reduced pressure and the residue dissolved in dichloromethane and washed with saturated $\mathrm{NaHCO}_{3}$ and water, dried $\left(\mathrm{MgSO}_{4}\right)$, filtered and the solvent removed in vacuum. The residue was chromatographed on a silica gel column as indicated.

3.1.28. 4-Benzyl-2-(N,N-diethylaminocarbonyloxymethyl)-1-(1H-indole-2-ylcarbonyl)piperazine (16a). Compound 16a was prepared using $13(300 \mathrm{mg}, 0.838 \mathrm{mmol})$, benzaldehyde $(101.6 \mu \mathrm{L}), \mathrm{NaBH}(\mathrm{OAc})_{3} \quad(263 \mathrm{mg})$ in dichloroethane $(50 \mathrm{~mL})$ and obtained as a white solid (380 mg, 93\%) after a silica gel column chromatography using $\mathrm{CH}_{2} \mathrm{Cl}_{2}$ as eluent: $R_{\mathrm{f}} 0.43\left(\mathrm{CH}_{2} \mathrm{Cl}_{2} / \mathrm{MeOH}, 95: 5\right.$, $\mathrm{v} / \mathrm{v}) ; \mathrm{mp} 74^{\circ} \mathrm{C}$; IR $\left(v \mathrm{~cm}^{-1}\right) 3270(\mathrm{NH}), 3067(\mathrm{ArCH})$, 1694 ( $\mathrm{C}=\mathrm{O}$ carbamate), $1603 \quad(\mathrm{C}=\mathrm{O}$ amide $), 1526$ $(\mathrm{ArC}=\mathrm{C}) ;{ }^{1} \mathrm{H} \mathrm{NMR}\left(\mathrm{CDCl}_{3}, \delta\right): 9.37$ (br s, $\left.1 \mathrm{H}, \mathrm{NH}\right)$, 7.25 (d, $1 \mathrm{H}, J=7.9 \mathrm{~Hz}, \mathrm{ArH}), 7.51-7.20$ (m, 7H, ArH,
ArH), 7.13 (d, 1H, $J=7,9 \mathrm{~Hz}, \mathrm{ArH}), 6.86$ (s, 1H, ArH), 4.10-5.20 (m, 4H, $\mathrm{CH}_{2} \mathrm{OC}=\mathrm{O}$ and piperazine), 3.57 $(\mathrm{d}, 1 \mathrm{H}, J=13.13 \mathrm{~Hz}, \mathrm{CHPh}), 3.47(\mathrm{~d}, 1 \mathrm{H}, J=13.13 \mathrm{~Hz}$, $\mathrm{CHPh}), 3.40,2.50\left(2 \mathrm{~m}, 6 \mathrm{H}, \mathrm{NCH}_{2} \mathrm{CH}_{3}\right.$ and piperazine), $2.00,2.40$ (m, 2H, piperazine), 0.60-1.20 (m, 6H, $\left.\mathrm{NCH}_{2} \mathrm{CH}_{3}\right) ;{ }^{13} \mathrm{C} \mathrm{NMR}\left(\mathrm{CDCl}_{3}, \delta\right): 163.45(\mathrm{C}=\mathrm{O}$ amide$)$, $154.94(\mathrm{C}=\mathrm{O}$ carbamate $), 136.30,136.15,127.54,126.97$, $131.54,130.13,129.14,124.60,121.81,120.52,111.95$, $106.29(\mathrm{ArC}=\mathrm{C}), 61.20\left(\mathrm{CH}_{2} \mathrm{OC}=\mathrm{O}\right), 60.94\left(\mathrm{PhCH}_{2} \mathrm{~N}\right)$, 51.57, $49.00\left(\mathrm{CH}_{2}\right.$ piperazine $), 41.74,41.05\left(\mathrm{NCH}_{2} \mathrm{CH}_{3}\right)$, 13.58, $13.00\left(\mathrm{NCH}_{2} \mathrm{CH}_{3}\right)$. Anal. $\left(\mathrm{C}_{26} \mathrm{H}_{32} \mathrm{O}_{3} \mathrm{~N}_{4} \cdot 0.1 \mathrm{H}_{2} \mathrm{O}\right)$ $\mathrm{C}, \mathrm{H}, \mathrm{N}$.

3.1.29. 2-(N,N-Diethylaminocarbonyloxymethyl)-1-(1Hindole-2-ylcarbonyl)-4-(3,4,5-trimethoxybenzyl)piperazine (16b). Compound $\mathbf{1 6 b}$ was prepared using 13 (330 mg, $0.92 \mathrm{mmol}), \quad 3,4,5$-trimethoxybenzaldehyde $(216 \mathrm{mg}), \mathrm{NaBH}(\mathrm{OAc})_{3}(292.5 \mathrm{mg})$ in dichloroethane $(50 \mathrm{~mL})$ and obtained as a white solid (440 mg, 95\%) after a silica gel column chromatography using $\mathrm{MeOH} / \mathrm{CH}_{2} \mathrm{Cl}_{2}\left(1: 99\right.$, v/v) as eluent: $R_{\mathrm{f}} 0.27\left(\mathrm{CH}_{2} \mathrm{Cl}_{2}\right.$ l $\mathrm{MeOH}, 95: 5, \mathrm{v} / \mathrm{v}) ; \mathrm{mp} 75^{\circ} \mathrm{C}$; IR $\left(v \mathrm{~cm}^{-1}\right) 3296(\mathrm{NH})$, 2967 (ArCH), 1689 ( $\mathrm{C}=\mathrm{O}$ carbamate), $1594 \quad(\mathrm{C}=\mathrm{O}$ amide), $1523(\mathrm{ArC}=\mathrm{C}) ;{ }^{1} \mathrm{H} \mathrm{NMR}\left(\mathrm{CDCl}_{3}, \delta\right): 9.90(\mathrm{br}$ s, $1 \mathrm{H}, \mathrm{NH}), 7.61(\mathrm{~d}, 1 \mathrm{H}, J=8 \mathrm{~Hz}, \operatorname{ArH}), 7.14$ (d, 1H, $J=8 \mathrm{~Hz}, \operatorname{ArH}), 7.25(\mathrm{~m}, 1 \mathrm{H}, \mathrm{ArH}), 7.13(\mathrm{~m}, 1 \mathrm{H}$, $\mathrm{ArH}), 6.85$ (s, 1H, ArH), 6.57 (s, 2H, ArH), 4.10-5.20 $\left(\mathrm{m}, 4 \mathrm{H}, \quad \mathrm{CH}_{2} \mathrm{OC}=\mathrm{O}\right.$ and piperazine $), 3.83(\mathrm{~s}, 9 \mathrm{H}$, $\left.\mathrm{OCH}_{3}\right), 3.57$ (d, $\left.1 \mathrm{H}, J=13.13 \mathrm{~Hz}, \mathrm{CH}_{2} \mathrm{Ar}\right), 3.28$ (d, $\left.1 \mathrm{H}, \quad J=13.13 \mathrm{~Hz}, \quad \mathrm{CH}_{2} \mathrm{Ar}\right), \quad 2.80-3.20 \quad(\mathrm{~m}, \quad 7 \mathrm{H}$, $\mathrm{NCH}_{2} \mathrm{CH}_{3}$ and piperazine), 2.00-2.50 (m, 2H, piperazine $), 0.98,0.83\left(2 \mathrm{~m}, 6 \mathrm{H}, \quad \mathrm{NCH}_{2} \mathrm{CH}_{3}\right) ;{ }^{13} \mathrm{C} \mathrm{NMR}$ $\left(\mathrm{CDCl}_{3}, \delta\right): 163.06(\mathrm{C}=\mathrm{O}$ amide $), 155.47(\mathrm{C}=\mathrm{O}$ carbamate), 153.16, 138.00, 135.79, 133.50, 129.20, 127.33, $124.20,121.78,120.32,111.72,105.60,105.22(\mathrm{ArC}=\mathrm{C})$, $62.77\left(\mathrm{CH}_{2} \mathrm{OC}=\mathrm{O}\right), 62.52\left(\mathrm{ArCH}_{2} \mathrm{~N}\right), 60.75, \quad 56.03$ $\left(\mathrm{CH}_{3} \mathrm{O}\right), \quad 53.40 \quad\left(\mathrm{CH}_{2}\right.$ piperazine $), \quad 41.03, \quad 39.00$ $\left(\mathrm{NCH}_{2} \mathrm{CH}_{3}\right), \quad 13.40, \quad 13.19 \quad\left(\mathrm{NCH}_{2} \mathrm{CH}_{3}\right)$. Anal. $\left(\mathrm{C}_{29} \mathrm{H}_{38} \mathrm{O}_{6} \mathrm{~N}_{4}\right) \mathrm{C}, \mathrm{H}, \mathrm{N}$.

3.1.30. 1-Benzyl-2-(N,N-diethylaminocarbonyloxymethyl)-4-(1H-indole-2-ylcarbonyl)piperazine (17a). Compound 17a was prepared using 15 (200 $\mathrm{mg}, 0.55 \mathrm{mmol})$, benzaldehyde $(78 \mu \mathrm{L}), \mathrm{NaBH}(\mathrm{OAc})_{3}(208 \mathrm{mg})$ in dichloromethane $(40 \mathrm{~mL})$ and obtained as a white solid (150 mg, 65\%) after a silica gel column chromatography using $\mathrm{CH}_{2} \mathrm{Cl}_{2}$ as eluent: $R_{\mathrm{f}} 0.43\left(\mathrm{CH}_{2} \mathrm{Cl}_{2} / \mathrm{MeOH}, 95: 5\right.$, v/ $\mathrm{v})$; mp $145^{\circ} \mathrm{C}$; IR $\left(v \mathrm{~cm}^{-1}\right) 3277(\mathrm{NH}), 3058(\mathrm{ArCH})$, $1696(\mathrm{C}=\mathrm{O}$ carbamate), 1603 ( $\mathrm{C}=\mathrm{O}$ amide), 1526 $(\mathrm{ArC}=\mathrm{C}) ;{ }^{1} \mathrm{H} \mathrm{NMR}\left(\mathrm{CDCl}_{3}, \delta\right): 9.09$ (br s, $\left.1 \mathrm{H}, \mathrm{NH}\right)$, $7.60(\mathrm{~d}, 1 \mathrm{H}, J=7.7 \mathrm{~Hz}, \mathrm{ArH}), 7.43-7.20(\mathrm{~m}, 7 \mathrm{H}$, $\mathrm{ArH}), 7.14(\mathrm{~d}, 1 \mathrm{H}, J=7.7 \mathrm{~Hz}, \mathrm{ArH}), 6.78(\mathrm{~s}, 1 \mathrm{H}$, $\mathrm{ArH}), 4.70-4.10\left(\mathrm{~m}, 4 \mathrm{H}, \mathrm{CH}_{2} \mathrm{OC}=\mathrm{O}\right.$ and piperazine $)$, $4.08(\mathrm{~d}, 1 \mathrm{H}, J=13.43 \mathrm{~Hz}, \mathrm{CHPh}), 3.66-3.51(\mathrm{~m}, 2 \mathrm{H}$, $\left.\mathrm{NCH}_{2} \mathrm{CH}_{3}\right), 3.46(\mathrm{~d}, 1 \mathrm{H}, J=13.43 \mathrm{~Hz}, \mathrm{CHPh}), 3.24$, $2.32\left(2 \mathrm{~m}, 7 \mathrm{H}, \mathrm{NCH}_{2} \mathrm{CH}_{3}\right.$ and piperazine), $0.60,1.20$ $\left(\mathrm{m}, 6 \mathrm{H}, \mathrm{NCH}_{2} \mathrm{CH}_{3}\right) ;{ }^{13} \mathrm{C} \mathrm{NMR}\left(\mathrm{CDCl}_{3}, \delta\right): 162.65$ $(\mathrm{C}=\mathrm{O}$ amide $), \quad 155.37 \quad(\mathrm{C}=\mathrm{O}$ carbamate $), 138.26$, $135.74,129.11,127.35,128.71,128.33,127.16,124.21$, $\begin{array}{lll}121.67, & 120.40, \quad 111.78, \quad 105.09 \quad(\mathrm{ArC}=\mathrm{C}), \quad 62.67\end{array}$ $\left(\mathrm{CH}_{2} \mathrm{OC}=\mathrm{O}\right), \quad 58.40 \quad\left(\mathrm{PhCH}_{2} \mathrm{~N}\right), 49.43,48.10 \quad\left(\mathrm{CH}_{2}\right.$ piperazine), 41.64, $41.16\left(\mathrm{NCH}_{2} \mathrm{CH}_{3}\right), 13.88,13.44$ $\left(\mathrm{NCH}_{2} \mathrm{CH}_{3}\right)$. Anal. $\left(\mathrm{C}_{26} \mathrm{H}_{32} \mathrm{O}_{3} \mathrm{~N}_{4}\right) \mathrm{C}, \mathrm{H}, \mathrm{N}$. 
3.1.31. 2-(N,N-Diethylaminocarbonyloxymethyl)-4-(1 Hindole-2-ylcarbonyl)-1-(3,4,5-trimethoxybenzyl)piperazine (17b). Compound 17b was prepared using 15 (180 $\mathrm{mg}, \quad 0.50 \mathrm{mmol}), \quad 3,4,3$-trimethoxybenzaldehyde $(117.8 \mathrm{mg}), \mathrm{NaBH}(\mathrm{OAc})_{3}(139 \mathrm{mg})$ in dichloroethane $(40 \mathrm{~mL})$ and obtained as a white solid $(200 \mathrm{mg}, 86 \%)$ after a silica gel column chromatography using $\mathrm{MeOH} / \mathrm{CH}_{2} \mathrm{Cl}_{2}(1: 99, \mathrm{v} / \mathrm{v})$ as eluent: $R_{\mathrm{f}} 0.38\left(\mathrm{CH}_{2} \mathrm{Cl}_{2} /\right.$ $\mathrm{MeOH}, 95: 5, \mathrm{v} / \mathrm{v}) ; \mathrm{mp} 148^{\circ} \mathrm{C}$; IR $\left(v \mathrm{~cm}^{-1}\right) 3310(\mathrm{NH})$, $2986(\mathrm{ArCH}), 1679(\mathrm{C}=\mathrm{O}$ carbamate $), 1595(\mathrm{C}=\mathrm{O}$ amide), $1526(\mathrm{ArC}=\mathrm{C}) ;{ }^{1} \mathrm{H} \mathrm{NMR}\left(\mathrm{CDCl}_{3}, \delta\right): 9.72(\mathrm{br}$ s, $1 \mathrm{H}, \mathrm{NH}), 7.59(\mathrm{~d}, 1 \mathrm{H}, J=8 \mathrm{~Hz}, \mathrm{ArH}), 7.40(\mathrm{~d}, 1 \mathrm{H}$, $J=8 \mathrm{~Hz}, \operatorname{ArH}), 7.24(\mathrm{~m}, 1 \mathrm{H}, \operatorname{ArH}), 7.10(\mathrm{~m}, 1 \mathrm{H}$, $\mathrm{ArH}), 6.78(\mathrm{~s}, 1 \mathrm{H}, \mathrm{ArH}), 6.58(\mathrm{~s}, 2 \mathrm{H}, \mathrm{ArH}), 4.54-4.10$ $\left(\mathrm{m}, 4 \mathrm{H}, \mathrm{CH}_{2} \mathrm{OC}=\mathrm{O}\right.$ and piperazine $), 3.99(\mathrm{~d}, 1 \mathrm{H}$, $\left.J=13 \mathrm{~Hz}, \mathrm{NCH}_{2} \mathrm{Ar}\right), 3.86\left(\mathrm{~m}, 9 \mathrm{H}, \mathrm{OCH}_{3}\right), 3.84-3.70$ (m, $3 \mathrm{H}$, piperazine), $3.43\left(\mathrm{~d}, 1 \mathrm{H}, J=13 \mathrm{~Hz}, \mathrm{NCH}_{2} \mathrm{Ar}\right)$, $3.30,3.36\left(\mathrm{~m}, 5 \mathrm{H}, \mathrm{NCH}_{2} \mathrm{CH}_{3}\right.$ and piperazine), 2.86, $2.38(2 \mathrm{~m}, 3 \mathrm{H}$, piperazine $), 1.06\left(\mathrm{~m}, 6 \mathrm{H}, \mathrm{NCH}_{2} \mathrm{CH}_{3}\right)$; ${ }^{13} \mathrm{C}$ NMR $\left(\mathrm{CDCl}_{3}, \delta\right): 162.73(\mathrm{C}=\mathrm{O}$ amide $), 155.35$ $(\mathrm{C}=\mathrm{O}$ carbamate $), 153.17,137.00,135.74,133.88$, $129.00,127.33,124.23,121.65,120.40,111.78,105.46$, $105.10 \quad(\mathrm{ArC}=\mathrm{C}), \quad 62.46 \quad\left(\mathrm{CH}_{2} \mathrm{OC}=\mathrm{O}\right), \quad 60.79, \quad 56.06$ $\left(\mathrm{CH}_{3} \mathrm{O}\right), 58.73(\mathrm{CH}$ piperazine $), 58.67\left(\mathrm{NCH}_{2} \mathrm{Ar}\right)$, $49.37\left(\mathrm{CH}_{2}\right.$ piperazine), 41.84, $41.17\left(\mathrm{NCH}_{2} \mathrm{CH}_{3}\right)$, 13.97, $13.34\left(\mathrm{NCH}_{2} \mathrm{CH}_{3}\right)$. Anal. $\left(\mathrm{C}_{29} \mathrm{H}_{38} \mathrm{O}_{6} \mathrm{~N}_{4}\right) \mathrm{C}, \mathrm{H}, \mathrm{N}$.

3.1.32. $2-(\mathrm{N}, \mathrm{N}$-Diethylaminocarbonyloxymethyl)-4-(1 Hindole-3-ylmethyl)-1-(3,4,5-trimethoxybenzoyl)piperazine (18). Compound 18 was prepared using $2(540 \mathrm{mg}$, $1.32 \mathrm{mmol})$, indole-3-carboxaldehyde $(211 \mathrm{mg})$, $\mathrm{NaBH}(\mathrm{OAc})_{3}(392 \mathrm{mg})$ in dichloroethane $(60 \mathrm{~mL})$ and obtained as a white powder (510 mg, 76\%) after a silica gel column chromatography using $\mathrm{MeOH} / \mathrm{CH}_{2} \mathrm{Cl}_{2} \quad(1: 99, \quad \mathrm{v} / \mathrm{v})$ as eluent: $R_{\mathrm{f}} \quad 0.39$ $\left(\mathrm{MeOH} / \mathrm{CH}_{2} \mathrm{Cl}_{2}, \quad 5: 95, \mathrm{v} / \mathrm{v}\right) ; \mathrm{mp} 75^{\circ} \mathrm{C}$; IR $(v$ $\left.\mathrm{cm}^{-1}\right) 3320(\mathrm{NH}), 3054(\mathrm{ArCH}), 1691(\mathrm{C}=\mathrm{O}$ carbamate), $1626 \quad(\mathrm{C}=\mathrm{O}$ amide $), 1584 \quad(\mathrm{ArC}=\mathrm{C}) ;{ }^{1} \mathrm{H}$ NMR $\left(\mathrm{CDCl}_{3}, \delta\right): 8.30$ (br s, $\left.1 \mathrm{H}, \mathrm{NH}\right), 7.73(\mathrm{~d}$, $1 \mathrm{H}, J=7 \mathrm{~Hz}, \operatorname{ArH}), 7.40(\mathrm{~d}, 1 \mathrm{H}, J=7 \mathrm{~Hz}, \operatorname{ArH})$, 7.06-7.30 (m, 3H, ArH), $6.50(\mathrm{~s}, 2 \mathrm{H}, \operatorname{ArH}), 5.30-$ $4.00\left(\mathrm{~m}, 2 \mathrm{H}, \mathrm{CH}_{2} \mathrm{OC}=\mathrm{O}\right), 3.83\left(\mathrm{~s}, 9 \mathrm{H}, \mathrm{OCH}_{3}\right)$, 3.74-3.46 (m, 3H, $\mathrm{NCH}_{2} \mathrm{CH}_{3}$ and piperazine), $3.40,2.00\left(2 \mathrm{~m}, 9 \mathrm{H}, \mathrm{NCH}_{2} \mathrm{CH}_{3}, \mathrm{CH}_{2}\right.$ indole and piperazine), $1.04\left(\mathrm{~m}, 6 \mathrm{H}, \mathrm{NCH}_{2} \mathrm{CH}_{3}\right) ;{ }^{13} \mathrm{C} \mathrm{NMR}$ $\left(\mathrm{CDCl}_{3}, \delta\right): 170.43(\mathrm{C}=\mathrm{O}$ amide $), 155.57 \quad(\mathrm{C}=\mathrm{O}$ carbamate), $139.00, \quad 136.44, \quad 131.17, \quad 127.39$, $111.79,123.50,122.58,121.93,119.46,119.37$, 111.04, $\quad 104.36 \quad(\mathrm{ArC}=\mathrm{C}), \quad 62.66 \quad\left(\mathrm{CH}_{2} \mathrm{OC}=\mathrm{O}\right)$, 60.77, $56.10\left(\mathrm{CH}_{3} \mathrm{O}\right), 53.84\left(\mathrm{CH}_{2}\right.$ indole $), 53.11$ $\left(\mathrm{CH}_{2}\right.$ piperazine $), 41.75,41.13\left(\mathrm{NCH}_{2} \mathrm{CH}_{3}\right), 13.84$, $13.48\left(\mathrm{NCH}_{2} \mathrm{CH}_{3}\right)$. Anal. $\left(\mathrm{C}_{29} \mathrm{H}_{38} \mathrm{O}_{6} \mathrm{~N}_{4}\right) \mathrm{C}, \mathrm{H}, \mathrm{N}$.

\subsection{Biological methods}

3.2.1. Platelet aggregation. The inhibition of platelet aggregation was conducted according to the published procedures. ${ }^{18}$ Briefly, it was determined using plateletrich plasma (PRP) of New Zealand rabbits by the method of Cazenave et al. ${ }^{28}$ Blood samples were collected from auricular artery into a citrate buffer (3.8\%, pH 7.4), and PRP was obtained by centrifugation for $15 \mathrm{~min}$ at $1200 \mathrm{rpm}$. The antagonists were sol- ubilized in DMSO and added to the incubated mixture and stirred PRP for $2 \mathrm{~min}$ before PAF $(2.5 \mathrm{nM})$ challenge. Platelet aggregation induced by PAF in the presence of the antagonists was monitored by continuous recording of light transmission in a dual-channel recorder (Cronolog Coultronics Apparatus) and was compared to a control aggregation induced by PAF alone. The drug concentration required to produce $50 \%$ inhibition $\left(\mathrm{IC}_{50}\right)$ was calculated from one dose-response curve.

3.2.2. Antiviral assay and cytotoxicity. All experiments were performed in triplicate in cells isolated from one blood donor. Antiviral assays and data analysis were conducted according to the published procedures. ${ }^{18,19}$ HIV-1 replication was assessed in cell culture supernatants by quantifying reverse transcriptase (RT), using the RetroSys ${ }^{\circledR}$ kit (Innovagen).

The cytotoxicity of different molecules was tested using a MTT (methyltetrazolium salt) assay (Invitrogen).

\subsection{Computational tools}

All the calculations were performed with the Gaussian $98^{25}$ program on a SGI Origin 3800. All the selected conformations were fully optimized within RHF molecular orbital formalism, using the $6-31 \mathrm{G}^{27}$ basis set. The $3 \mathrm{D}$ electrostatic potential has also been computed at that level with the same basis set.

\section{Acknowledgments}

We thank Karen Naissant-Storck for her technical assistance. This work was supported by the Scientific Council of the Universite Paris 7 - Denis Diderot (Paris, France). Georges Dive is chercheur qualifié of the Fond National de la Recherche Scientifique (FNRS), Brussels. This work was supported by the Belgian program on Interuniversity Poles of Attraction initiated by the Belgian State, Prime Minister's Office, Service fédéraux des affaires scientifiques, techniques et culturelles (PAI No. P5/33).

\section{Supplementary data}

Supplementary data associated with this article can be found, in the online version, at doi:10.1016/j.bmc. 2006.07.043.

\section{References and notes}

1. An, S. F.; Groves, M.; Gray, F.; Scaravilli, F. J. Neuropathol. Exp. Neurol. 1999, 58, 1156.

2. Davis, L. E.; Hjelle, B. L.; Miller, V. E.; Palmer, D. L.; Llewellyn, A. L.; Merlin, T. L.; Young, S. A.; Mills, R. G.; Wachsman, W.; Wiley, C. A. Neurology 1992, 42, 1736.

3. McArthur, J. C.; Hoover, D. R.; Bacellar, H.; Miller, E. N.; Cohen, B. A.; Becker, J. T.; Graham, N. M.; 
McArthur, J. H.; Selnes, O. A.; Jacobson, L. P. Neurology 1993, 43, 2245.

4. Bacellar, H.; Munoz, A.; Miller, E. N.; Cohen, B. A.; Besley, D.; Selnes, O. A.; Becker, J. T.; McArthur, J. C. Neurology 1994, 44, 1892.

5. Brodt, H. R.; Kamps, B. S.; Gute, P.; Knupp, B.; Staszewski, S.; Helm, E. B. AIDS 1997, 11, 1731.

6. Sacktor, N.; Lyles, R. H.; Skolasky, R.; Kleeberger, C.; Selnes, O. A.; Miller, E. N.; Becker, J. T.; Cohen, B.; McArthur, J. C. Neurology 2001, 56, 257.

7. Maschke, M.; Kastrup, O.; Esser, S.; Ross, B.; Hengge, U.; Hufnagel, A. J. Neurol. Neurosurg. Psychiatry 2000, 69, 376.

8. Sacktor, N.; McDerrmott, M. P.; Merder, K.; Schifitto, G.; Selnes, O. A.; McArthur, J. C.; Stern, Y.; Albert, S.; Palumbo, D.; Kieburtz, K.; De Marcaida, J. A.; Cohen, B.; Epstein, L. J. Neurovirol. 2002, 8, 136.

9. Gray, F.; Chretien, F.; Vallat-Decouvelaere, A. V.; Scaravilli, F. J. Neuropathol. Exp. Neurol. 2003, 62, 429.

10. Cherner, M.; Masliah, E.; Ellis, R. J.; Marcotte, T. D.; Moore, D. J.; Grant, I.; Heaton, R. K. Neurology 2002, 59, 1563.

11. Gonzalez-Scarano, F.; Martin-Garcia J. Nat. Rev. Immunol. 2005, 5, 69 .

12. Enting, R. H.; Hoetelmans, R. M.; Lange, J. M.; Burger, D. M.; Beijnen, J. H.; Portegies, P. AIDS 1998, 12, 1941.

13. Nath, A. J. Infect. Dis. 2002, 186(Suppl. 2), 193.

14. Gelbard, H. A.; Nottet, H. S. L.; Swindells, S.; Jett, M.; Dzenko, K. A.; Genis, P.; White, R.; Wang, L.; Choi, Y.B.; Zhang, D.; Lipton, S. A.; Tourtelotte, W. W.; Epstein, L. G.; Gendelman, H. E. J. Virol. 1994, 68, 4628.

15. Perry, S. W.; Hamilton, J. A.; Tjoelker, L. W.; Dbaibo, G.; Dzenko, K. A.; Epstein, L. G.; Hannun, Y.; Whittaker, J. S.; Dewhurst, S.; Gelbard, H. A. J. Biol. Chem. 1998, 273, 17660 .

16. Locardi, C.; Petrini, P.; Boccoli, G.; Testa, U.; Dieffenbach, C.; Butto, S.; Belardelli, F. J. Virol. 1990, 64, 5874.

17. Weissman, D.; Poli, G.; Bousseau, A.; Fauci, A. S. Proc. Natl. Acad. Sci. U.S.A. 1993, 90, 2537.

18. Serradji, N.; Bensaid, O.; Martin, M.; Kan, E.; Dereuddre-Bosquet, N.; Redeuilh, C.; Huet, J.; Heymans, F.;
Lamouri, A.; Clayette, P.; Dong, C. Z.; Dormont, D.; Godfroid, J. J. J. Med. Chem. 2000, 43, 2149.

19. Serradji, N.; Martin, M.; Bensaid, O.; Cisternino, S.; Rouselle, C.; Dereuddre-Bosquet, N.; Huet, J.; Redeuilh, C.; Lamouri, A.; Dong, C. Z.; Clayette, P.; Scherrmann, J. M.; Dormont, D.; Heymans, F. J. Med. Chem. 2004, 47, 6410 .

20. Serradji, N.; Bensaid, O.; Martin, M.; Sallem, W., Dereuddre-Bosquet, N.; Benmehdi, H.; Lamouri, A.; Dive, G.; Clayette, P.; Heymans, F. Bioorg. Med. Chem. 2006, accepted.

21. Yongxin, R.; Chari, R. V. J. U.S. Patent 6,534,660, 2003.

22. Wang, J.; Le, N.; Heredia, A.; Song, H.; Redfield, R.; Wang, L. X. Org. Biomol. Chem. 2005, 3, 1781.

23. Boger, D. L.; Patel, M. J. Med. Chem. 1987, 52, 3934.

24. Lamouri, A.; Heymans, F.; Tavet, F.; Dive, G.; Batt, J. P.; Blavet, N.; Godfroid, J. J. J. Med. Chem. 1993, 36, 990.

25. Frisch, M. J.; Trucks, G. W.; Schlegel, H. B.; Scuseria, G. E.; Robb, M. A.; Cheeseman, J. R.; Zakrzewski, V. G.; Montgomery, Jr. J. A.; Stratmann, R. E.; Burant, J. C.; Dapprich, S.; Millam, J. M.; Daniels, A. D.; Kudin, K. N.; Strain, M. C.; Farkas, O.; Tomasi, J.; Barone, V.; Cossi, M.; Cammi, R.; Mennucci, B.; Pomelli, C.; Adamo, C.; Clifford, S.; Ochterski, J.; Petersson, G. A.; Ayala, P. Y.; Cui, Q.; Morokuma, K.; Malick, D. K.; Rabuck, A. D.; Raghavachari, K.; Foresman, J. B.; Cioslowski, J.; Ortiz, J. V.; Baboul, A. G.; Stefanov, B. B.; Liu, G.; Liashenko, A.; Piskorz, P.; Komaromi, I.; Gomperts, R.; Martin, R. L.; Fox, D. J.; Keith, T.; Al-Laham, M. A.; Peng, C. Y.; Nanayakkara, A.; Gonzalez, C.; Challacombe, M.; Gill, P. M. W.; Johnson, B.; Chen, W.; Wong, M. W.; Andres, J. L.; Gonzalez, C.; Head-Gordon, M.; Replogle, E. S.; Pople, J. A. Gaussian 98, Revision A.7, Gaussian, Inc., Pittsburgh PA, 1998.

26. Batt, J. P.; Lamouri, A.; Tavet, F.; Heymans, F.; Dive, G.; Godfroid, J. J. J. Lipid Mediat. 1991, 4, 343.

27. Hehre, W. J.; Ditchfield, R.; Pople, J. A. J. Chem. Phys. 1972, 56, 2257.

28. Cazenave, J. P.; Benveniste, J.; Mustard, J. F. Lab. Invest. 1979, 41, 275. 